Щ्)FRANÇAISE

$>\mathrm{DE}$

필 PÉDAGOGIE

\section{Revue française de pédagogie}

Recherches en éducation

199 | avril-mai-juin 2017

L'histoire des disciplines : un champ de recherche en mutation

\title{
Une ségrégation peut en cacher une autre. La répartition des élèves entre classes à prendre au sérieux
}

The need to distinguish between segregation at both class and school level. The need to take the distribution of students between classes seriously

Julien Danhier, Émilie Martin, Alejandra Alarcon-Henriquez, Rob Kaelen et Dirk Jacobs

\section{OpenEdition}

Journals

Édition électronique

URL : https://journals.openedition.org/rfp/6109

DOI : 10.4000/rfp.6109

ISSN : 2105-2913

Éditeur

ENS Éditions

Édition imprimée

Date de publication : 30 juin 2017

Pagination : 117-138

ISBN : 979-10-362-0108-0

ISSN : 0556-7807

Référence électronique

Julien Danhier, Émilie Martin, Alejandra Alarcon-Henriquez, Rob Kaelen et Dirk Jacobs, « Une ségrégation peut en cacher une autre. La répartition des élèves entre classes à prendre au sérieux», Revue française de pédagogie [En ligne], 199 | avril-mai-juin 2017, mis en ligne le 30 juin 2020, consulté le 06 janvier 2022. URL : http://journals.openedition.org/rfp/6109 ; DOI : https://doi.org/10.4000/rfp. 6109 


\title{
Une ségrégation peut en cacher une autre. La répartition des élèves entre classes à prendre au sérieux
}

\author{
Julien Danhier \\ Émilie Martin \\ Alejandra Alarcon-Henriquez \\ Rob Kaelen \\ Dirk Jacobs
}

\begin{abstract}
La Fédération Wallonie-Bruxelles (Belgique) est profondément marquée par une ségrégation académique et socio-économique qui prend source dans une organisation particulière du système éducatif. La ségrégation entre écoles a été largement mise en évidence. La ségrégation entre classes au sein des écoles n’a été que peu abordée par la littérature, mais constitue pourtant un phénomène notable. Premièrement, les apports de différentes mesures et données mobilisées pour mesurer la ségrégation entre écoles sont présentés. Deuxièmement, un indice provenant de l'analyse multiniveau est proposé pour mesurer simultanément la ségrégation entre écoles et au sein des écoles. Il est appliqué à un échantillon de 10309 élèves de deuxième année commune (enseignement secondaire ordinaire) dans 591 classes et 97 écoles de la FWB. Les analyses montrent une ségrégation importante, notamment entre classes au sein des écoles.
\end{abstract}

Mots-clés (TESE) : environnement social (société, culture, langues et religion), analyse statistique, classe, système éducatif

\section{Introduction}

La recherche a montré que la Fédération WallonieBruxelles ${ }^{1}$ (FWB) est profondément marquée par une

1 Depuis 1989, la Belgique est composée de trois systèmes éducatifs autonomes reflétant la division du pays en communautés linguistiques. Dans cet article, nous nous centrons sur la «Communauté française de Belgique » qui scolarise environ $44 \%$ des élèves belges et est compétente pour l'enseignement francophone en Wallonie et à Bruxelles (MCF, 2010). Dans le texte, le vocable «Fédération Wallonie-Bruxelles» est utilisé pour la désigner tandis que le vocable «Flandre» est utilisé pour désigner la «Communauté flamande de Belgique». 
ségrégation académique ${ }^{2}$ et socio-économique (Baye, Demeuse, Nicaise et al., 2005). L'importance du rôle joué par les structures a également été soulignée (Monseur \& Demeuse, 2001 ; Demeuse \& Friant, 2010) : l'organisation de l'offre scolaire en quasi-marché et I'organisation de carrières différenciées concourent au regroupement d'élèves socialement et académiquement similaires dans des écoles très différentes les unes des autres. Cette composition différentielle est problématique dès lors qu'elle a un effet sur la réussite scolaire des élèves (Dumay \& Dupriez, 2008) ou sur leurs aspirations (Dupont \& Lafontaine, 2011). Preuve de l'intérêt que le phénomène suscite, le gouvernement de la FWB a identifié, dans son contrat pour l'éducation (MCF, 2005), la ségrégation comme l'un des quatre problèmes majeurs de son enseignement et y fait de son slogan «Non aux écoles ghettos» I'une des 10 priorités. Le texte fait d'ailleurs référence à deux mesures pour définir des objectifs concrets : «ramener la part de variance des performances des élèves attribuable à l'établissement de $56 \%$ à $40 \%$; réduire le niveau de ségrégation scolaire [sur la base de l'origine sociale] à moins de $40 \%$ ». Une série de décrets visant à réguler les inscriptions en première secondaire cristallise depuis lors les débats relatifs à la ségrégation scolaire.

Si la ségrégation entre écoles a été largement étudiée, c'est moins le cas de la ségrégation au sein des écoles. Il est toutefois capital d'étudier ce niveau. Une régulation plus intense des mécanismes à l'origine de la ségrégation entre écoles pourrait mener à un déplacement de ces mécanismes entre classes au sein des écoles et donc à une amplification de la ségrégation entre classes, c'est-à-dire à un niveau où prennent davantage place une série d'effets (effets de pairs et effets des enseignants) permettant de comprendre la manière dont la ségrégation agit sur les performances scolaires et la construction de relations sociales. Les analyses statistiques se rapportant à ce niveau manquent pourtant cruellement en FWB. Cette ségrégation requiert en fait des informations souvent absentes des bases de données. Les recherches menées en dehors de la FWB nous permettent toutefois d'envisager l'ampleur du phénomène. Dans certains départements français par exemple, une ségré-

2 Dans le présent article, nous distinguerons la ségrégation "académique» basée sur des mesures de réussite scolaire de la ségrégation "socio-économique» basée sur leur origine gation relativement importante a été récemment mesurée entre classes (Riegert \& Ly, 2016).

Durant le premier semestre de l'année scolaire 2014-2015, notre équipe a conduit une large collecte parmi les écoles de la FWB. Cette collecte nous a permis d'observer une ségrégation non négligeable au sein des écoles au début de l'enseignement secondaire. L'intérêt d'examiner la question au niveau de la seconde année secondaire réside également dans l'existence, en Fédération Wallonie-Bruxelles, d'un tronc commun censé durer jusqu'à la fin de cette année. L'observation d'une importante ségrégation à ce niveau questionne donc l'existence effective de ce tronc commun.

L'article se structure comme suit : après avoir présenté les structures scolaires responsables de la ségrégation en Belgique francophone, nous mettrons en évidence les apports respectifs de différentes mesures statistiques mobilisées dans ce contexte et ce qu'elles nous apprennent sur la ségrégation. Si cette revue partielle de la littérature est centrée sur la FWB, il ne s'agit pas de minorer l'énorme travail fourni par les chercheurs du monde entier, mais plutôt de synthétiser les nombreux travaux qui ont pu être menés dans un contexte scolaire particulier. Nous proposerons ensuite une méthode probante pour distinguer la part de la ségrégation attribuable aux classes ou aux écoles et en discuterons les résultats.

\section{Une organisation ségréguante}

Nous définirons la ségrégation comme la séparation, dans des lieux (ou des moments) d'enseignement distincts, d'étudiants porteurs de caractéristiques différemment valorisées par la société (Delvaux, 2005). Les ségrégations d'un système éducatif sont des caractéristiques qui prennent leur source dans un modèle d'organisation et des structures spécifiques conduisant à regrouper des élèves aux caractéristiques scolaires ou non scolaires similaires. Demeuse et Baye (2008) ont montré que les systèmes dont les structures déploient des outils pour séparer les élèves ont tendance à être plus ségrégués ${ }^{3}$. Ces structures peuvent toutefois être très diverses. Il est donc nécessaire de

3 Les auteurs ont classé les pays selon une multitude d'indicateurs mesurant les ségrégations notamment académiques et socio-économiques et selon les structures déployées pour sépare les élèves. Ils observent un lien modéré entre les rangs occupés par les pays sur ces deux dimensions (rho de Spearman de 0,46 sur PISA 2003). 
Figure 1. Organisation de l'enseignement de la Fédération Wallonie-Bruxelles
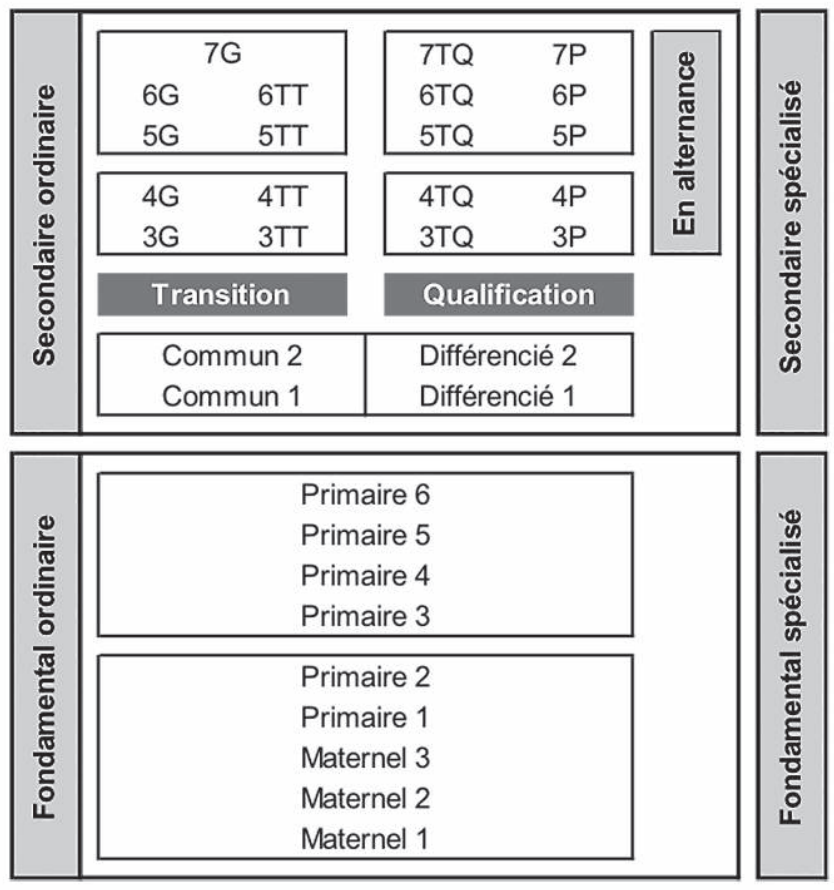

considérer une multitude d'indicateurs, notamment les normes de passage d'une année à l'autre, l'usage ou non du redoublement et la part des élèves ainsi maintenus, l'âge de la première orientation et les effectifs de chaque filière, la proportion d'élèves dans l'enseignement spécialisé, les possibilités de choix en matière d'inscriptions scolaires ou encore le regroupement en classes d'âge ou de niveau (Monseur \& Demeuse, 2001).

Dans les pages qui suivent, nous détaillerons deux ensembles de règles interdépendantes qui contribuent à séparer les élèves et donnent une configuration particulière au système de la FWB. Les premières différencient les carrières scolaires et les secondes traitent de l'appariement des élèves à leur école.

\section{Stratifications de la carrière scolaire}

La figure 1 représente le système éducatif de la FWB selon deux axes : I'axe horizontal où l'orientation est le principal outil pour séparer les étudiants dans des filières différentes (de l'enseignement général vers l'enseignement professionnel, mais également l'enseignement en alternance et l'enseignement spécialisé) et l'axe vertical où le redoublement sépare les élèves au cours de leur progression à travers les années d'études. Ces axes correspondent à ce qui peut se concevoir comme des stratifications du système éducatif (OECD, 2014). L'orientation et le redoublement, en tant qu'outils interdépendants de gestion de l'hétérogénéité et de sélection sociale, participent à la même figure (Crahay, 2000). Ils ont un effet cumulatif assignant les élèves en échec à des places spécifiques dans la hiérarchie scolaire et les mettant en retard par rapport à ceux qui se sont maintenus dans la trajectoire scolaire la plus courte et la plus exigeante. Notons que le redoublement prend place à la fois avant et après I'orientation (Delvaux, 2000), soit pour maintenir I'élève sans l'orienter soit pour compléter l'orientation déjà mobilisée. Cette accumulation est massive en FWB puisqu'à la fin du secondaire, ils sont $60 \%$ à avoir au moins un an de retard (MCF, 2016).

Formellement, la carrière scolaire d'un élève s'inscrit dans un continuum pédagogique en trois étapes qui s'étend de l'entrée en maternelle à la seconde année de l'enseignement secondaire (jusqu'à l'âge de 14 ans, environ). Concrètement cependant, plusieurs affaiblissements de ce tronc commun existent. À côté de l'enseignement ordinaire, un enseignement spécialisé accueille environ $5 \%$ des élèves. De plus, un enseignement dit "différencié», s'adressant aux élèves 
n'ayant pas obtenu le diplôme requis en fin de primaire, accueille $6 \%$ des élèves du premier degré du secondaire (la troisième étape du continuum pédagogique). En résumé, à ce moment de la scolarité, plus de $10 \%$ des élèves ne suivent plus le tronc commun. L'orientation dans des filières séparées commence en troisième année de l'enseignement secondaire, ce dernier accueillant $48 \%$ des élèves dans une filière qualifiante (MCF, 2016). Comme dans de nombreux systèmes éducatifs, cette stratification horizontale est organisée sous forme d'une hiérarchie où la trajectoire académique (la section de transition et plus particulièrement l'enseignement général) est le cadre de référence pour les enseignants (André, 2011) et les élèves (Ferrara \& Friant, 2014). Cette stratification prend en outre la forme d'une sélection sociale puisqu'à niveau équivalent, les élèves de milieu défavorisé se retrouvent davantage dans les filières les moins prestigieuses (Boone \& Van Houtte, 2013 en Flandre).

Les règles définissant la progression de l'élève à travers les années d'études successives constituent l'axe vertical de la gestion de l'hétérogénéité. C'est le redoublement qui est l'outil le plus largement utilisé en FWB. II y apparaît comme une pratique fonctionnelle au regard de ses fonctions latentes, à savoir la gestion de l'hétérogénéité, le contrôle sur les élèves, la définition de la réputation de l'école et le maintien de l'autonomie professionnelle de l'enseignant (Draelants, 2006), bien que son efficacité pédagogique soit discutée (Crahay, 2007 ; Draelants, 2018). La FWB apparaît comme l'un des systèmes éducatifs avec la plus grande proportion d'élèves ayant redoublé à la fois dans le primaire (Martin, Mullis \& Foy, 2011) et dans le secondaire où les élèves de 15 ans sont $48 \%$ à déclarer avoir déjà redoublé (OECD, 2014). Les proportions de doubleurs par année d'étude sont légèrement supérieures à $3 \%$ en primaire, mais dépassent les $15 \%$ dans certaines années de l'enseignement secondaire (MCF, 2016). Bien que des réformes successives aient tenté d'en limiter l'usage (en interdisant le redoublement à la fin de certaines années d'études puis en définissant un continuum pédagogique formé de trois étapes où une seule "année complémentaire» est disponible à chaque étape, si besoin), les tendances historiques soulignent son ancrage puisque chaque limitation du redoublement est rapidement compensée par sa plus large utilisation à un autre endroit de la carrière scolaire.

\section{Quasi-marché scolaire}

Comme le précise l'article 24 de la Constitution belge, l'enseignement est libre. Cet article assure, d'une part, à chaque individu (ou association) de pouvoir organiser une école, et d'autre part, à chaque parent de pouvoir choisir l'école fréquentée par son enfant. Bien qu'historiquement cette liberté de choix portait sur la possibilité de choisir entre écoles confessionnelles et non confessionnelles, elle s'apparente aujourd'hui à une logique consumériste dont la visée est le choix de la meilleure école (Maroy \& Dupriez, 2000). L'enseignement est également défini comme gratuit. Les écoles peuvent ainsi espérer un subventionnement public. Concrètement, ce subventionnement est en fonction du nombre d'élèves que l'école accueille. Une telle combinaison (subventionnement public «par tête» et libre choix de l'école) a reçu le vocable de «quasi-marché » (Le Grand, 1991), une institution hybride qui tend à combiner à la fois le contrôle des usagers potentiels qui peuvent choisir entre les écoles et le contrôle de l'administration publique qui les subventionne (Vandenberghe, 1996).

Cette structuration du système en marchés (plus ou moins) locaux constitue un cadre cognitif puissant pour les familles et les écoles puisqu'elle oriente fortement leurs pratiques (Devleeshouwer \& Rea, 2011). En effet, dans chaque espace local, les familles choisissent l'école qu'elles pensent la plus adaptée pour leur enfant. Alors que la proximité joue un rôle dans ce choix, sont également déterminants d'autres facteurs tels que la réputation de l'école, sa composition, les programmes proposés (aux différentes étapes de la carrière scolaire) ainsi que la place perçue de l'enfant dans la hiérarchie scolaire. Les écoles, quant à elles, sont en compétition les unes avec les autres et mettent en œuvre des stratégies pour attirer non seulement un nombre d'élèves suffisant pour maintenir ou développer leurs ressources, mais encore des élèves dont la qualité est associée à la réputation de l'école et à ses conditions de travail (Delvaux \& Joseph, 2006; Maroy \& van Zanten, 2009). Ces stratégies prennent place en début, mais également à chaque étape de la carrière scolaire où les écoles sélectionnent leur public, certaines écrémant leur public et d'autres le récupérant (Demeuse, Derobertmasure, Friant et al., 2007). Les écoles se spécialisent pour accueillir la diversité des profils d'étudiants, créant ainsi des effets dits de niche. La faible régulation des inscriptions et la faible pression mise sur les écoles pour respecter les règles en vigueur leur permettent de sélectionner leurs élèves à la fois sur des bases académiques mais également 
socio-économiques, et ce d'autant plus lorsque la demande excède le nombre de places disponibles. Une conséquence visible de cette structuration est une certaine adéquation entre le public scolarisé et le type d'école qui le scolarise. L'offre des écoles et les carrières scolaires des élèves étant différenciées, le quasi-marché peut ainsi se définir comme l'expression spatiale de la différenciation des carrières scolaires. Ces caractéristiques concourent à favoriser un système scolaire ségrégué et à créer différents types d'écoles dont les écoles dites «ghetto » ou « sanctuaire » constituent l'expression extrême (Demeuse \& Friant, 2010).

Une série d'études qui se sont attachées à mettre en regard les flux entre lieux de résidence et de scolarisation des élèves soulignent le rôle des structures scolaires. Avec des méthodes diverses (notamment cartographiques) et sur la base des données administratives disponibles (et de leurs limites), cette approche défend l'hypothèse selon laquelle la ségrégation scolaire en FWB reflète partiellement la ségrégation résidentielle, mais ne s'y réduit certainement pas. Ces études font le constat d'une diversité importante entre élèves d'un même quartier, d'un recrutement des écoles qui y est socialement différencié et d'une mobilité importante entre les lieux de domicile et de scolarisation. Particulièrement en milieu urbain, si les écoles proches ne diffèrent pas de beaucoup au regard de l'origine de leurs élèves et proposent des offres parfois similaires, les familles, quel que soit leur milieu d'origine, peuvent choisir et choisissent effectivement entre plusieurs écoles, même dans l'enseignement maternel ou primaire où le recrutement est réputé plus local (Delvaux, 2003; Delvaux \& Serhadlioglu, 2014; Marissal, 2014). L'offre scolaire locale structure la mobilité des élèves (y compris défavorisés) dans leur rencontre d'une offre adaptée à leur parcours scolaire (Danhier \& Devleeshouwer, 2017). Les données administratives ne permettent pourtant pas de modéliser correctement les choix des parents, et le critère de proximité semble rester un critère déterminant lorsqu'il s'agit de prédire I'allocation des élèves à leur école (Friant, 2015).

Assumant que le choix libre de l'école est l'un des paramètres contribuant à la ségrégation, le Gouvernement de la FWB a tenté de réguler, pour la première fois en 2008-2009, les inscriptions en première année de l'enseignement secondaire, mais a également limité les changements d'écoles en cours de cycles ${ }^{4}$. Une période

4 Depuis 2010-2011, le décret en vigueur au moment de l'écriture met en place une gestion centralisée des demandes. Dans les éta- d'agitation a suivi, avec la promulgation d'un nouveau décret chaque année et comme conséquence des conflits entre et au sein des acteurs de la société civile et des partis politiques, mais aussi d'une mauvaise réception par une partie de l'opinion publique ayant interprété ces mesures comme une réduction inacceptable de leur liberté de choix (Delvaux \& Maroy, 2009; Ryelandt, 2013). En ajoutant un objectif de déségrégation à un décret visant initialement la régulation des inscriptions excédentaires, le législateur est à l'origine d'une confusion entre ces objectifs et d'un renforcement de leur caractère polémique qui s'est largement répandu dans les médias et l'opinion publique. Sur le plan de la déségrégation, les décrets sont toutefois relativement modestes puisqu'ils se bornent à traiter les inscriptions et plus particulièrement les inscriptions excédentaires dans le nombre limité d'écoles que cela concerne, et ce uniquement en première année de l'enseignement secondaire. En avril 2012, seuls 6,5 \% des élèves de première n'étaient pas assurés d'avoir une place dans l'école de leur premier choix l'année suivante (MCF, 2014). Si la question des décrets régulant les inscriptions est loin d'épuiser la question de la ségrégation en FWB, il est toutefois important de comprendre qu'ils définissent un cadre de référence prégnant pour cette thématique. De plus, si le changement de composition de certains établissements se traduit par une intensification de la ségrégation entre classes, il pourra expliquer un déplacement de la ségrégation en FWB.

\section{La mesure de la ségrégation en Fédération Wallonie-Bruxelles}

Divers indices ont été utilisés en FWB pour mesurer la ségrégation (voir tableau 2 en annexe). Les mesures présentées ci-dessous se limitent aux ségrégations académique et socio-économique, ce qui est cohérent

blissements avec un excédent de demandes, les élèves sont classés selon un indice composite calculé à partir des préférences exprimées, de critères géographiques (distances entre le domicile, l'école primaire et l'école secondaire) et pédagogiques. Les élèves classés en ordre utile sont inscrits dans l'école de leur premier choix. Notons que les élèves issus d'écoles à indice socio-économique faible sont prioritaires pour un cinquième des places ( $d$ 'autres priorités sont également définies, comme le fait d'avoir un frère ou une sœur dans l'école ou un parent travaillant dans l'école). Lorsque l'école est complète, l'administration se réserve environ $20 \%$ des places disponibles à attribuer sur base d'un algorithme (dit «algorithme d'acceptation différée») visant à maximiser les préférences des parents. 
avec la tradition en FWB où le gouvernement concentre ses actions sur la base des critères scolaires et sociaux, à l'inverse de la Flandre où l'on fait largement référence aux critères linguistiques et ethniques (Jacobs \& Rea, 2005). Préalablement, il est utile de présenter brièvement les deux principales sources de données utilisées de manière récurrente. Ces deux sources de données présentent des qualités et des faiblesses notables qui conditionnent les analyses possibles et leurs portées.

Les données administratives fournissent une première source. Depuis 2004-2005, au 15 janvier de chaque année, l'administration conserve dans son "Comptage des élèves » (CMP) à des fins de gestion la liste exhaustive de ses élèves, leur position précise dans le système scolaire et leur lieu de résidence. $\mathrm{Si}$ aucune mesure de la performance scolaire n'y est disponible, un indice socio-économique (ISE) l'est néanmoins. En effet, dans le cadre des discriminations positives, un indice synthétique (couvrant les revenus, les qualifications, les conditions de vie et l'emploi) a été calculé pour chaque secteur statistique (la plus petite subdivision territoriale en Belgique) et attribué aux élèves selon leur lieu de résidence (Demeuse, 2002). Bien que cet indice ait été utilisé de façon extensive, il présente certaines carences notables. Tout d'abord, il ne s'agit pas d'un indice individuel. Comme les secteurs accueillent des populations hétérogènes, la réduction artificielle de la variance due à l'utilisation d'un indice agrégé peut introduire des biais lors de la mesure de la ségrégation (Delvaux, 2003). Ensuite, certains indicateurs ne sont pas disponibles à ce niveau le plus désagrégé, datent de plus de 10 ans et sont faiblement corrélés avec l'indice.

La seconde source de données provient de collectes internationales telles qu'organisées par l'OCDE (PISA) ou I'IEA (TIMSS, PIRLS). La FWB a participé aux éditions successives de PISA, de PIRLS depuis 2006 (en quatrième primaire uniquement) et à l'édition de TIMSS en 1995 (en $2^{\mathrm{e}}$ secondaire). Nous renvoyons le lecteur aux rapports officiels de ces enquêtes pour de plus amples informations. Il est toutefois important de rappeler que les deux organisations mesurent finement les performances scolaires des élèves (modèles de réponse à l'item en lecture, mathématiques et sciences pour PISA, mathématiques et sciences pour TIMSS et lecture pour PIRLS). De plus, les deux organisations ont choisi un échantillon stratifié en deux étapes, où les écoles sont d'abord sélectionnées proportionnellement à leur taille. L'OCDE a, pour sa part, sein de chaque école, alors que l'IEA a sélectionné une ou deux classes complètes. Enfin, les deux organisations ont inclus dans leurs collectes une mesure individuelle de l'origine socio-économique calculée à partir du plus haut niveau d'éducation et de la plus haute profession parmi les deux parents (HISEI) ainsi que des possessions domestiques et culturelles.

Tout comme le nombre de sources, le nombre d'indices mobilisés en FWB est limité. Un premier indice célèbre est l'indice de Dissimilarité (Duncan \& Duncan, 1955). Cet indice D varie entre 0 et 1 (pour une ségrégation maximale) et représente la proportion d'élèves désavantagés à "déplacer sans remplacement» pour atteindre une égale répartition des élèves avantagés et désavantagés entre les écoles (Cortese, Falk \& Cohen, 1976). Un deuxième indice s'inscrit dans la lignée de deux projets (soutenu par la Commission européenne) visant à comparer les systèmes éducatifs européens, menés de 2001 à 2005 par le GERESE (Groupe européen de recherche sur l'équité des systèmes éducatifs). Les chercheurs ont opté pour un indice (GS), historiquement nommé Delta (Duncan, 1961), et pouvant être interprété comme la proportion d'élèves désavantagés qui devraient être "échangés» pour atteindre une égale répartition de ces élèves entre les écoles. L'indice GS est en fait directement dérivable de l'indice D (multiplication par l'inverse de la proportion d'élèves du groupe désavantagé) et a d'ailleurs été utilisé pour en faciliter l'interprétation (Cortese, Falk \& Cohen, 1976). L'usage de l'indice GS a été ardemment défendu par Gorard, membre de l'équipe partenaire du GERESE ayant en charge la production de l'indice de ségrégation (Gorard \& Taylor, 2002), qui le préférait à l'indice de Dissimilarité pour sa forte invariance compositionnelle. Concrètement, l'indice GS serait un choix avantageux puisqu'il ne varie pas lorsqu'une partie des élèves avantagés passent dans le groupe des élèves désavantagés tant que leur répartition entre les écoles reste la même. Toutefois, à la différence de l'indice D, l'indice GS n'est pas symétrique (sa valeur diffère pour le groupe désavantagé et le groupe avantagé) et n'est pas borné à 1 (mais à l'inverse de la proportion de désavantagés). Notons enfin que les deux indices ne varient pas lorsque des élèves désavantagés changent entre deux écoles dont les proportions d'élèves désavantagés sont toutes deux en dessous ou au-dessus de la moyenne (James \& Taeuber, 1985 ; Gorard \& Taylor, 2002).

Dupriez et Vandenberghe (2004) ainsi qu'Hindriks et Lamy (2013) ont appliqué l'indice D aux données PISA. En 2014, c'est cet indice qui a été utilisé par le 
ministère (MCF, 2014) pour évaluer l'évolution de la ségrégation en $1^{\text {re }}$ secondaire à la suite des décrets régulant les inscriptions. Cette évaluation a permis de montrer que la ségrégation diminue très légèrement au cours de la période étudiée et présente une valeur très faible qui peut s'expliquer par le choix de l'année d'étude (uniquement la première année commune de l'enseignement secondaire) et de la variable (composition sociale du quartier de résidence, et non origine de l'élève lui-même). L'indice GS a logiquement été mobilisé dans les rapports des projets précédemment mentionnés et les travaux des chercheurs y ayant collaboré (Baye, Demeuse, Nicaise et al., 2005; Baye, Demeuse, Monseur et al., 2006; Baye \& Demeuse, 2008), mais a également permis de chiffrer l'objectif de déségrégation (inférieur à 0,40 ) dans le contrat stratégique pour l'éducation (MCF, 2005).

Ces différentes mobilisations nous inspirent deux commentaires. Premièrement, les intervalles de confiance sont rarement fournis pour les mesures effectuées sur les collectes internationales. Si l'on considère que la quantification de l'incertitude est une composante essentielle de l'analyse quantitative, ce manque nous semble problématique ${ }^{5}$. Certains auteurs ont toutefois fourni des intervalles. Jenkins, Micklewright et Schnepf (2008) ont utilisé les centiles de la méthode "bootstrap» (échantillon aléatoire simple avec remplacement au niveau des écoles) pour calculer un intervalle de confiance à leur indice $D$ sur un échantillon fusionné des données PISA 2000 et 2003. Suivant cette approche, la ségrégation socio-économique apparaît comme significativement plus importante en Belgique que dans les pays scandinaves ou aux États-Unis. Hindriks, Verschelde, Rayp et alii (2009) ont utilisé la même méthode sur les données PISA 2006 et montrent que la ségrégation socio-économique plus élevée de la FWB ne se distingue pas significativement de celle de la Flandre. Il n'est toute-

5 L'analyse quantitative est par essence empreinte d'erreurs. Une tâche fondamentale consiste à minimiser ces erreurs lorsque c'est possible et sinon à les identifier et les quantifier. Nous pouvons relever, entre autres, les erreurs de mesure, de traitement, de non-réponse, d'échantillonnage ou de couverture, qui requièrent chacune un traitement spécifique (Groves, Floyd, Couper et al., 2009). Selon que l'erreur est aléatoire ou systématique, la mesure sera moins précise ou davantage biaisée. Le risque lié à chaque type d'erreur dépend de la source utilisée. La quantification de l'erreur d'échantillonnage est en enjeu central lors de l'utilisation de collectes internationales alors que l'utilisation de l'indice socio-économique du comptage est soumise à un risque important d'erreur de mesure. Les non-réponses concernent, quant à elles, les deux types de sources précédemment cités. fois pas garanti que l'utilisation d'une méthode bootstrap n'imitant pas le plan d'échantillonnage de la collecte puisse garantir la validité des inférences. Danhier, Jacobs, Devleeshouwer et alii (2014) ont utilisé les poids de rééchantillonnage (BRR) disponibles dans PISA pour calculer un intervalle de confiance normal en postulant que GS, comme $D$, sont normalement distribués (Ransom, 2000). Si la ségrégation académique est significativement plus élevée qu'en moyenne au sein des pays de l'OCDE, ce n'est pas le cas de la ségrégation socio-économique qui est néanmoins significativement plus élevé en FWB qu'en Finlande, Norvège ou Suède.

Secondement, ces indices recourent à un seuil arbitraire pour rendre dichotomique la variable d'intérêt et définir un groupe de référence (les désavantagés), ce qui peut poser plusieurs problèmes. Tout d'abord, le choix d'un seuil plutôt qu'un autre peut conduire à des conclusions différentes. Danhier et Friant (2016) ont comparé l'évolution de la ségrégation (indice GS) des élèves de 15 ans en Belgique de 2006 à 2012. Sur la base des éditions successives de PISA, la ségrégation diminue de 2009 à 2015, quel que soit le seuil choisi, mais cette diminution est parfois significative, parfois non significative (les intervalles de confiance sont calculés grâce aux poids de rééchantillonnage). Sur le CMP par contre, la conclusion change selon le seuil choisi. Ainsi la ségrégation augmente-t-elle lorsque le premier décile est choisi comme seuil et diminue-t-elle lorsque c'est le premier quintile qui est choisi, ce dernier scénario ayant été choisi pour l'évaluation des décrets du ministère (MCF, 2014). Par conséquent, si les conclusions sont liées au choix du seuil, il devient difficile de comparer les résultats des mesures dont le seuil est différent. Plus généralement, la catégorisation d'une variable continue est une pratique déconseillée en analyse quantitative, car elle modifie les relations entre variables et a des conséquences négatives en termes de taille d'effets, de puissance et de fiabilité (MacCallum, Zhang, Preacher et al., 2002).

L'indice dit « racine carrée » (Hutchens, 2001) a l'avantage d'être décomposable. Cette propriété permet d'observer si la ségrégation se situe entre des sous-groupes ou en leur sein. Concrètement, cet indice a permis de vérifier si l'importante ségrégation observable en Belgique est imputable à l'existence de filières différentes (Verschelde, Hindriks, Rayp et al., 2010). Alors que 50,9\% [IC 43,$5 ; 57,8$ ] de la ségrégation se situent entre les filières en Flandre, seuls 25,6\% [IC 13,1;34,5] s'y situent en FWB. En d'autres termes, si l'organisation de filières 
participe de manière non négligeable à la ségrégation, on observe d'importantes différences de public entre les écoles d'une même filière. Notons que les auteurs ont également appliqué cette méthode à la segmentation du système belge en réseaux d'enseignement concurrents. En fait, la majeure partie des écoles sont subsidiées par l'administration centrale, mais sont organisées soit par cette administration, soit par des autorités territoriales locales, soit par des associations privées. Cette segmentation et la concurrence qui en résulte sont souvent pointées comme l'une des sources de la ségrégation en FWB, mais selon les auteurs, seuls 6,6\% de la ségrégation se situent entre réseaux, un pourcentage qui n'est pas significativement différent de 0 .

Une approche alternative permet de calculer la ségrégation à partir de variables continues et se base sur la partition de leur variance. Lorsqu'on recourt à I'ANOVA ou aux analyses multiniveaux, il est courant de diviser la variance de la variable dépendante selon qu'elle est imputable aux individus ou à leur appartenance à un niveau de regroupement (l'école, par exemple). Techniquement, la proportion de la variance entre les écoles (ICC pour corrélation intraclasse ou VPC pour composante de la partition de la variance) est utilisée comme indice de ségrégation (White, 1986; Willms \& Paterson, 1995). Plus la proportion de la variance attribuable au niveau de regroupement est importante, plus le système sera ségrégué, c'est-à-dire constitué de classes ou d'écoles aux valeurs très diverses, mais dont les populations sont homogènes.

Ce type d'approche a été appliquée aux données 1981 et 1991 de l'IEA (Grisay, 1995; Lafontaine, 1996). Ces auteures soulignent deux observations. Premièrement, la ségrégation sur la base des résultats scolaires semble plus prononcée que la ségrégation sur base sociale. Secondement, l'indice appliqué aux résultats scolaires est bien plus limité en primaire qu'en secondaire, un constat similaire à celui pouvant être fait à partir de l'indice GS sur la base des données TIMSS 1995 et PISA 2000 (Baye, Demeuse, Nicaise et al., 2005). Sous le vocable d'indice d'inclusion sociale ou académique, le VPC a été utilisé dans les rapports successifs de I'OCDE (OECD, 2004, 2007, 2010, 2014). En 2005, il fournit l'un des deux indices permettant de chiffrer le contrat stratégique pour l'éducation (MCF, 2005). Sur la base de I'ISE du CMP de 2008, Danhier et Deny (2009) ont observé une ségrégation importante en FWB qui ne peut être réduite à des disparités régionales (si la région Bruxelles-Capitale présente une
FWB dans son ensemble, elle est toutefois moindre, mais non négligeable, dans les différentes provinces wallonnes). Danhier et Friant (2016) ont toutefois observé une surestimation systématique de l'indice observée sur le CMP par rapport à l'indice calculé sur PISA qui peut s'expliquer par une réduction de la variance intra-école due à l'utilisation d'un indice socio-économique agrégé. À nouveau, peu d'études fournissent des intervalles de confiance, excepté la dernière où les auteurs ont eu recours à une méthode dite de «bootstrap».

Un intérêt de l'utilisation du VPC réside dans son lien direct avec la modélisation multiniveau ${ }^{6}$. Lafontaine et Monseur (2011) ont décomposé la variance des performances scolaires entre écoles belges (PISA 2006) selon qu'elle est expliquée par l'origine sociale de l'élève, son retard scolaire ou sa filière. Si l'origine sociale présente un effet unique s'élevant à $5 \%$, elle présente surtout un effet joint avec le retard et la filière qui atteint les $16 \%$. Les effets uniques du retard et de la filière valent respectivement $23 \%$ et $21 \%$. En d'autres termes, la variance entre écoles est davantage expliquée par des différences en termes de parcours scolaire. De plus, le recrutement social différentiel des écoles semble traduire majoritairement un recrutement sélectif sur la base des résultats scolaires. Le constat est similaire en 2009 (Danhier \& Martin, 2014).

Pour autant, la technique et son application aux données internationales ne sont pas sans limites. Premièrement, la partition de la variance des performances scolaires traduit un recrutement différentiel, mais également, une progression différentielle des élèves selon les écoles et classes. À mesure que la date

6 L'analyse de régression multiniveau (ou hiérarchique) est une famille d'analyses développées pour tenir compte des agglomérats présents dans les données, soit parce qu'ils existent dans la réalité, soit parce qu'ils sont créés par le chercheur lors de sa collecte. Les élèves regroupés dans des classes, elles-mêmes regroupées dans des écoles fournissent un exemple typique de données dites «hiérarchiques». Sans être exhaustives, deux qualités de ce type d'analyse méritent d'être soulignées. Premièrement, lorsque ces agglomérats sont présents dans les données, les observations ne peuvent pas être considérées comme indépendantes (des élèves fréquentant une même classe ou une même école évoluent dans un même contexte scolaire et ont donc tendance à avoir un profil plus similaire que des élèves provenant d'écoles ou de classes différentes). Cette relation doit être statistiquement prise en compte dans l'analyse sous peine d'obtenir des résultats faussement significatifs et l'analyse multiniveau est une des méthodes qui permettent de le faire. Deuxièmement, les caractéristiques, non seulement des individus, mais aussi des agglomérats, peuvent avoir une influence. L'analyse multiniveau permet de modéliser des variables à chaque niveau et d'observer leur influence selon le niveau considéré. 
d'administration s'éloigne de l'entrée dans l'école ou la classe, la progression différentielle peut peser davantage dans la partition de la variance. Deuxièmement, la définition des niveaux de l'analyse est capitale. En effet, lorsqu'un niveau intermédiaire est ignoré, la variance de ce niveau est distribuée entre les niveaux adjacents (Opdenakker \& Van Damme, 2000). Les auteurs ont pu montrer qu'au début de l'enseignement secondaire flamand, la variance des résultats en mathématiques située aux niveaux de la classe et de l'enseignant était redistribuée au niveau des élèves (environ $60 \%$ ) et des écoles (environ $40 \%$ ) lorsque les niveaux de la classe et de l'enseignant étaient ignorés. Par conséquent, l'absence du niveau classe conduit à une surestimation des différences interindividuelles et donc à une sous-estimation de la ségrégation totale, mais également à une surestimation de la ségrégation entre écoles. Troisièmement, le choix de l'unité définissant le niveau «école» a des effets sur la valeur de l'indice, un choix qui peut se porter sur l'établissement (une unité administrative), I'implantation (le lieu géographique) ou la filière au sein de l'école. De plus, ce choix pouvant différer selon les pays, il rend la comparaison difficile (Monseur \& Lafontaine, 2005). Quatrièmement, le design complexe doit être pris en compte. L'utilisation des pondérations reste notamment une question complexe pour l'analyse multiniveau (Pfeffermann, Skinner, Holmes et al., 1998).

Pour conclure sur la ségrégation entre écoles, nous pouvons avancer sans trop de risque que les indices précédemment présentés démontrent la présence d'une ségrégation importante en FWB et la placent systématiquement parmi les systèmes scolaires les plus ségrégués que ce soit en regard d'indicateurs relatifs à la réussite scolaire ou à l'origine socio-économique. II semblerait en outre que la ségrégation académique est plus marquée que la ségrégation sociale et qu'elle augmente au fur et à mesure de la carrière scolaire, en parallèle de l'intensification de la stratification scolaire et de l'effet différentiel des écoles. II semblerait encore qu'elle ne peut être réduite à des disparités géographiques ni à des disparités de recrutement entre réseaux d'écoles concurrents. De nombreux problèmes techniques persistent toutefois. Le choix des données reste déterminant, mais aucune des propositions n'est entièrement satisfaisante. Alors que les données administratives permettent d'observer finement les différences de ségrégation entre années d'études ou aires géographiques et d'en suivre l'évolution au cours du temps, elles manquent de mesures relatives à la réussite scolaire et d'une mesure de l'origine sociale individuelle. Les enquêtes internationales fournissent ces mesures, mais seulement pour certaines années d'études, par bonds de plusieurs années et sans possible distinction entre aires géographiques.

\section{Ségrégation entre classes}

Si la mesure de la ségrégation entre écoles a été abondamment traitée par la recherche quantitative, la mesure de la ségrégation entre classes au sein des écoles ne l'a été que rarement. Pourtant, si cette ségrégation entre classes est importante, une partie du phénomène risque de rester invisible, non seulement parce que la ségrégation entre classes pourrait participer substantiellement à ce phénomène, mais également parce qu'elle pourrait présenter une série de caractéristiques propres, non réductibles à la ségrégation entre écoles.

Si le cadre d'une organisation ségréguante peut s'appliquer largement à la ségrégation entre classes, une série de facteurs interne à l'école pourraient venir le renforcer. Le vécu de la ségrégation entre écoles et au sein des écoles pourrait également être fort différent. Au regard de son effet, la ségrégation entre classes apparaît spécifique dans la mesure où il s'agit d'un niveau où prennent davantage place une série d'effets (notamment des effets de pairs et des effets des enseignants) permettant de comprendre la manière dont la ségrégation agit sur les performances scolaires (Bressoux, 1995) et la construction de relations sociales. Dans cet article, lorsque nous parlons de ségrégation totale, nous référerons à la ségrégation des lieux de scolarisation dans son ensemble en ne dissociant pas les niveaux «école» ou «classe».

Dans la littérature internationale, des indices ont été mobilisés pour quantifier le phénomène et mettent en évidence la présence d'une ségrégation entre classes d'une même école. Comme l'indice D n'est pas sensible à la division des unités de regroupement et ne change donc pas lorsqu'une école est divisée en deux classes où la proportion d'élèves désavantagés est identique (Allen \& Vignoles, 2007), Kalogrides et Loeb (2013) ont comparé cet indice entre classes et écoles aux ÉtatsUnis. Selon l'année et le district considéré, la ségrégation sociale entre classes (sur la base d'une mesure dichotomique d'accès à des repas à prix réduit) compte pour 8 à $32 \%$ de la ségrégation totale (à savoir, la ségrégation entre classes et écoles). Toutefois, si l'indice D n'est pas 
sensible à la division des unités de regroupement, il n'est pas décomposable en éléments qui peuvent s'additionner (Frankel \& Volij, 2011). II semble donc qu'il ne soit pas adapté pour calculer la contribution des classes à la ségrégation totale. Ce n'est pas le cas de l'indice d'exposition (Bell, 1954) qui a l'avantage d'être décomposable et de fournir une interprétation simple, à savoir la probabilité qu'un élève désavantagé rencontre un élève avantagé dans sa classe. Cet indice a notamment été utilisé pour étudier la ségrégation ethnique. En 20002001, l'indice de ségrégation des Blancs et non-Blancs entre classes en fin de primaire à New York représente $6,3 \%$ de la ségrégation totale (Conger, 2005) tandis qu'il représente la moitié de la ségrégation totale dans l'enseignement secondaire en Caroline du Nord (Clotfelter, Ladd \& Vigdor, 2003).

En France, Duru-Bellat et Mingat (1997) ont observé que $21,4 \%$ de la variance du score initial des élèves des deux premières années du collège (données collectées de 1989 à 1992) se situe entre les classes au sein des établissements, alors que $13,3 \%$ de cette variance se situe entre établissements. En simulant une situation à plusieurs classes par établissements grâce à la présence de classe sur deux années adjacentes $\left(5^{\mathrm{e}}\right.$ et $4^{\mathrm{e}}$ année) dans les données TIMSS 1995, Beaton et O'Dwyer (2002) ont pu mesurer que $13,1 \%$ de la variance des résultats en mathématiques se situe entre les classes et 12,0\% entre les établissements. Ces deux mesures du VPC indiquent une ségrégation entre classes particulièrement importante. Récemment, Riegert et Ly (2016) ont calculé l'indice d'exposition pour mesurer la ségrégation sociale entre classes. En troisième secondaire et selon le département, cette ségrégation atteint entre 0,03 et 0,08 point, ce qui représente en moyenne $24,8 \%$ de la ségrégation totale dans les départements les plus ségrégués (entendre : dont la ségrégation totale dépasse 0,15 ). Ces exemples montrent que la ségrégation entre classes au sein des écoles n'est pas un phénomène négligeable.

Nous n'avons pas connaissance d'une telle approche en $\mathrm{FWB}$, ce qui est dommageable puisque cela mène à une sous-estimation de la ségrégation dans son ensemble d'autant plus importante qu'elle est présente entre classes. Ce manque est principalement dû à l'absence de bases de données qui permettent d'identifier la classe et l'école. Lorsqu'elles le permettent, le nombre de classes par écoles n'est pas suffisant pour pouvoir mesurer correctement la contribution de chaque niveau à la ségrégation totale, soit parce que la ment à cause du coût exorbitant), soit parce que la réalité ne s'y plie pas (si l'école n'organise qu'une seule classe pour l'année considérée ou si elle ne regroupe pas ses élèves en «classe»). Sur la base d'une collecte de données auprès des élèves de cinquième primaire (1980-1981), Grisay (1984) observe que 42,1 \% de la variance à un test de français se situe entre les classes (VPC), sans pourtant pouvoir distinguer la ségrégation entre classes et entre écoles. Sur la base des données PIRLS 2011, Danhier (2017) a calculé une ségrégation entre les classes de $0,37[0,33 ; 0,42]$ (les poids de rééchantillonnage «jackknife» ont été utilisés pour calculer un intervalle de confiance). Dans ces données, une seule classe a été sondée dans $41 \%$ des écoles, ce qui ne permet pas d'y distinguer la contribution propre du regroupement en classes au sein des écoles.

Le manque criant de mesures de la ségrégation entre classes en FWB justifie nos analyses. Nous faisons I'hypothèse que cette ségrégation n'est pas négligeable au début de l'enseignement secondaire et qu'elle entraîne une sous-estimation de la ségrégation totale. D'autres sous-hypothèses sont également explorées. Premièrement, les disparités socio-économiques entre aires géographiques sont perçues comme une limite forte à toute politique de déségrégation. Nous faisons toutefois l'hypothèse qu'une part importante de la ségrégation prend place à un niveau local et n'est donc pas réductible à des disparités régionales. Secondement, la sélection étant principalement académique, la ségrégation socio-économique pourrait n'être que le reflet de cette sélection. Nous faisons cependant l'hypothèse qu'il existe une ségrégation proprement socio-économique non réductible à des niveaux scolaires objectivables.

\section{Données et méthode}

Durant le premier semestre de l'année scolaire 20142015 , notre équipe a conduit une large collecte parmi les écoles de la FWB. Un test de performance en mathématiques et un questionnaire mesurant des attitudes et des caractéristiques socio-démographiques ont été administrés aux élèves. En fin d'année scolaire, les données collectées ont été fusionnées avec les résultats de l'épreuve communautaire standardisée (CE1D). Parmi les 492 implantations scolaires organisant une deuxième année commune (âge théorique des élèves: 13 ans), 164 écoles ont été sélectionnées sur la base d'un sondage à strates proportionnelles (quartiles socio-économiques, réseaux d'enseignement, taille de l'école et régions) et 
106 ont accepté de participer. Tous les élèves de deuxième année commune ont été invités à participer à notre enquête (les élèves de deuxième année différenciée n'ont pas été sondés). Une fois les absences, les refus et les différentes erreurs comptabilisés, 10395 élèves dans 598 classes et 103 écoles ont été effectivement atteints. Traduits en taux de participation, $62,8 \%$ des écoles sélectionnées, $82,7 \%$ des élèves de ces écoles et 58,6 \% des élèves de notre base d'échantillonnage initiale font donc partie de notre échantillon 7 . Afin de pouvoir distinguer la ségrégation entre classes de la ségrégation entre écoles, les écoles n'organisant qu'une seule classe de deuxième et les classes où seul un élève de deuxième a participé à l'enquête ont été exclues ce qui nous laisse un échantillon final de 10309 élèves dans 591 classes et 97 écoles. Une école organise en moyenne 6 classes de deuxième et 17 élèves par classe ont, en moyenne, participé à notre enquête.

Afin de mesurer la ségrégation entre classes, nous aurons recours à la partition de la variance de l'analyse de régression linéaire multiniveau. Cette méthode a l'avantage de nous permettre de mesurer la ségrégation sur une variable continue (forme que prend, on va le voir, notre variable caractérisant l'origine socio-économique), de distinguer la part de la variance imputable à l'élève, à la classe et à l'école, mais également de modéliser certaines caractéristiques des élèves et écoles lorsque nous mesurons la ségrégation. En d'autres termes, nos indices de ségrégation mesurent la proportion de la variance totale qui est située au niveau des classes d'une part et des écoles d'autre part ${ }^{8}$. De plus, la distribution du VPC n'est pas connue. Afin de pouvoir produire la distribution postérieure de notre indice et y définir un intervalle de crédibilité couvrant $95 \%$ de la distribution, la méthode de Monte-Carlo par chaînes de Markov (50 000 itérations) a été utilisée.

L'analyse multiniveau présente toutefois une série des contraintes. Premièrement, la taille de l'échantillon à chaque niveau doit être suffisante et est le plus souvent problématique aux niveaux «classe» et «école». Si la variance est correctement estimée dans la plupart des cas, la taille de son intervalle de confiance est sous-estimée lorsque le nombre de groupes est limité,

7 Malgré des taux de participation acceptables, nous ne pouvons exclure la possibilité d'une sous-estimation de la variance à l'un ou l'autre niveau du modèle. Aucune correction n'a toutefois été implémentée.

8 Les modèles utilisés permettent à la constante de varier à chaque niveau, mais les pentes des variables indépendantes sont fixées lorsque ces variables sont introduites dans les modèles. et le reste légèrement lorsque 100 groupes sont modélisés (Maas \& Hox, 2005). L'utilisation de la méthode de Monte-Carlo par chaînes de Markov avec une distribution préalable diffuse permet toutefois d'obtenir de bons résultats même lorsque le nombre de groupes est faible (Browne \& Draper, 2006). Dans le cas très spécifique de la mesure de la ségrégation, la faible taille des classes est également importante, car elle peut être la source d'une ségrégation aléatoire (Cortese, Falk \& Cohen, 1976). Cette ségrégation aléatoire entre classes a été simulée (100 fois) en redistribuant aléatoirement les élèves entre les classes d'une même école. La ségrégation aléatoire entre classes atteint en moyenne $0,3 \%$ avec un maximum de 0,9\%. Les résultats de nos analyses seront analysés en regard de ces valeurs plutôt qu'en regard d'un 0 théorique.

Deuxièmement, l'usage des pondérations reste un sujet complexe (Pfeffermann, Skinner, Holmes et al., 1998). Dans ce cas-ci, la méthode d'échantillonnage brièvement présentée ci-dessus a été choisie de sorte que nos analyses multiniveaux ne requièrent pas de pondérations. Aucune pondération n'a par ailleurs été introduite pour corriger les éventuels biais liés à la non-participation de certaines écoles et de certains élèves. Bien que les écoles du réseau de la FWB soient davantage représentées (au détriment de celles du réseau libre), ainsi que les écoles de taille moyenne (au détriment de celles de petite taille), nous avons décidé de ne pas pondérer notre échantillon, compte tenu de la bonne représentation des écoles sur base de leur composition socio-économique, comme de leur situation géographique, mais également par souci de maintenir nos analyses à un niveau de complexité raisonnable.

La recherche d'un indice de ségrégation unique pour rendre compte de la ségrégation sociale et les prérequis de la méthode choisie nous poussent à utiliser une construction factorielle mesurant l'origine socio-économique. Ce type de construction s'inscrit dans une tradition quantitativiste américaine (White, 1982). Sans entrer dans une description détaillée de ses nombreuses opérationnalisations ni des différentes théories expliquant son effet sur la réussite scolaire, nous nous contenterons d'observer les trois dimensions qui sont classiquement mobilisées pour sa construction, à savoir le niveau d'éducation des parents, l'activité professionnelle des parents et la richesse familiale (ou les biens et services à disposition). Nous acceptons cependant la critique selon laquelle une telle opérationnalisation est un affaiblissement du concept de capital, notamment culturel, de 
la théorie de la reproduction (Draelants \& Ballatore, 2015). Dans le cadre de cet article, le plus haut niveau d'éducation et la plus haute activité professionnelle des parents ont été utilisés en accord avec le modèle de dominance (Erikson, 1984). Les activités professionnelles ont été recodées selon la nomenclature ISCO 2008 et transformées en une variable continue (Ganzeboom, 2010). La richesse a été mesurée par une échelle recensant des biens et services dont jouit le ménage, supposément plus fiable comme mode de collecte auprès des jeunes élèves (Yang \& Gustafsson, 2004). Le modèle de réponse à l'item utilisé (unidimensionnel à deux paramètres) rassemble la possession d'un ordinateur et d'une chambre individuelle, la disponibilité d'un bureau, de plusieurs voitures et salles de bain, l'aide d'une femme de ménage ainsi que l'usage de l'avion pour voyager. La Belgique, comme la France, faisant partie d'un groupe de pays où l'activité professionnelle et le niveau d'éducation pèsent plus qu'ailleurs sur la réussite aux tests PISA 2009 (Keskpaik \& Rocher, 2011), mais où les biens culturels (la possession de littérature classique ou le nombre de livres disponibles à la maison) y ont également un poids important, le nombre de livres disponibles à la maison est donc ajouté à ces trois premières dimensions. Une analyse factorielle a été appliquée à ces quatre variables et fournit une solution factorielle acceptable au regard des indices d'adéquation aux données, mais plus limite au regard des saturations ${ }^{9}$. Bien que la corrélation s'élevant à 0,31 entre ce facteur et la réussite en mathématiques en début d'année soit significative et dans le sens attendu, tout doute quant à la validité d'un tel construit n'est pas écarté. II reste cependant que ce construit capturant (certes de manière imparfaite) la complexité de l'origine socio-économique

9 Concernant les indices d'adéquation du modèle aux données, Hu et Bentler (1999) ont suggéré de combiner le CFI, le SRMR et le RMSEA dont on attend qu'ils soient respectivement supérieur à 0,96 , inférieur à 0,09 et inférieur à 0,06 . L'intervalle de confiance du RMSEA permet en outre de rejeter I'hypothèse d'une faible adéquation aux données lorsque la borne supérieure de cet intervalle ne dépasse pas 0,08 (Hooper, Coughlan \& Mullen, 2008). Dans ce modèle, les indices d'adéquation sont supérieurs aux seuils recommandés (CFI de 0,99, SRMR de 0,01 et borne supérieure de l'intervalle de confiance du RMSEA à 0,06). Concernant les saturations, Tabachnick et Fidell (2007) rapportent la règle selon laquelle une saturation est excellente lorsqu'elle est supérieure à 0,7 et de qualité progressivement moindre en dessous de ce seuil (très bien jusque 0,63 , bien jusque 0,55 , juste jusque 0,45 ). Avec des saturations pour les livres, la richesse, le niveau d'éducation et l'activité professionnelle atteignant respectivement $0,48,0,54,0,66,0,71$, le modèle est fournit un outil essentiel dans la construction de notre indice de ségrégation.

Diverses variables de contrôle ont également été mobilisées. Le territoire étant socialement contrasté, une partie de la ségrégation entre écoles pourrait traduire des disparités régionales ou subrégionales. Les divisions administratives du territoire pouvant ne pas être pertinentes en FWB, des chercheurs ont proposé plusieurs scénarios pour identifier des bassins scolaires (Delvaux, Demeuse, Dupriez et al., 2005) sur la base d'une analyse des liens d'interdépendance entre zones de recrutement scolaire ${ }^{10}$. Les bassins sont introduits dans le modèle 1 sous forme de variables indicatrices. Deux variables proprement scolaires sont ensuite intégrées dans le modèle 2 . Le niveau en mathématiques a été estimé sur la base du pourcentage de réponses correctes à une épreuve standardisée administrée en début d'année scolaire et couvrant un curriculum similaire à l'épreuve standardisée du CE1D (Jacobs, Martin, Alarcon-Henriquez et al., 2017). Cette variable nous permet d'approcher la manière dont les élèves sont évalués à cette étape de leur parcours. Le retard scolaire additionne les redoublements et maintiens successifs que l'élève a subis au cours de sa carrière scolaire. Les résultats à l'épreuve servent également de variable dépendante dans le dernier modèle 3 pour comparer les ségrégations académique et sociale.

Une précision technique s'impose. Les variables mesurant l'origine socio-économique présentent un taux de non-réponse élevé, notamment lorsqu'il s'agit de collecter les activités professionnelles des parents. L'origine socio-économique est donc manquante pour $28 \%$ des élèves de notre échantillon. Avec une telle proportion de valeurs manquantes, I'utilisation d'un modèle d'imputation multiple est conseillée. Dans ce contexte, 50 imputations sont produites sur la base d'un modèle statistique incluant un ensemble de variables individuelles socio-démographiques et scolaires, mais également contextuelles (au niveau de la classe $)^{11}$. Les analyses ont ensuite été menées sur ces

10 Parmi les scénarios, nous avons choisi d'utiliser celui appelé "Petits bassins B» dont les limites en termes d'interdépendances sont moins claires et dont l'offre scolaire peut être incomplète, mais qui a l'avantage de découper le territoire en aires dont la taille ne pose pas de problèmes organisationnels (contrairement à d'autres découpages aux limites plus claires).

11 Un modèle d'imputation par équations chaînées (van Buuren \& Groothuis-Oudshoorn, 2011) a été utilisé dans le cadre de ces analyses. Se basant sur des analyses préparatoires, ce modèle inclut les variables suivantes, leur méthode individuelle d'imputation se trouvant entre parenthèses : les résultats en mathématiques en 


\begin{tabular}{lcccc}
\hline & $\begin{array}{c}\text { Ségrégation } \\
\text { entre écoles }\end{array}$ & $\begin{array}{c}\text { Ségrégation } \\
\text { entre classes }\end{array}$ & $\begin{array}{c}\text { Ségrégation } \\
\text { totale }\end{array}$ & $\begin{array}{c}\text { Part } \\
\text { de la classe }\end{array}$ \\
\hline Modèle 0 & $20,8[15,5 ; 26,0]$ & $5,5[4,2 ; 6,8]$ & $26,3[21,3 ; 31,2]$ & $21,1[14,5 ; 27,6]$ \\
\hline Modèle 1 & $17,4[12,3 ; 22,4]$ & $5,7[4,4 ; 7,0]$ & $23,1[18,3 ; 27,9]$ & $25,0[17,1 ; 33,0]$ \\
\hline Modèle 2 & $11,6[7,8 ; 15,4]$ & $4,2[3,0 ; 5,3]$ & $15,7[12,0 ; 19,5]$ & $26,8[17,8 ; 35,9]$ \\
\hline Modèle 3 & $26,8[20,7 ; 32,9]$ & $9,8[8,0 ; 11,6]$ & $36,6[31,2 ; 42,0]$ & $27,0[19,9 ; 34,1]$ \\
\hline
\end{tabular}

50 bases de données complètes et leurs résultats ont été combinés de manière à tenir compte de l'incertitude liée au manque d'une partie de l'information. La méthode utilisée pour produire les intervalles de confiances est précisée en annexe.

Les différentes analyses ont été effectuées sous $R$ avec les packages suivants : lavaan (pour l'analyse factorielle), Itm (pour les modèles de réponse à l'item), mice (pour les imputations multiples) et $\mathrm{r} 2 \mathrm{mlwin}$ (pour l'analyse multiniveau).

\section{Résultats}

Le tableau 1 présente les résultats de nos modèles successifs. Dans le modèle 0 , alors que la part de la variance de l'origine socio-économique entre classes au sein des écoles s'élève à 5,5\%, la part entre écoles atteint $20,8 \%$. La ségrégation totale (entre classes et écoles) s'élève donc à $26,3 \%$. Ce modèle souligne à nouveau l'importante ségrégation observée entre les écoles de la FWB. La ségrégation entre classes est de moindre

début et fin d'année, le retard scolaire, l'activité professionnelle des parents la plus prestigieuse, la richesse, le nombre de livres à la maison, l'origine socio-économique (multiniveau «élèves et classes»), le plus haut niveau d'éducation des parents, la langue parlée à la maison, la motivation des élèves, l'implication des parents (predictive mean matching), les redoublements antérieurs, le sexe, le statut sans emploi de la mère ou du père (régression logistique), les statut et origine migratoire des élèves (modèle logit multinomial) ainsi que la moyenne au niveau de la classe de l'occupation la plus prestigieuse, le nombre de livres à la maison, le plus haut niveau d'éducation des parents, la richesse, l'origine sociale et les résultats en mathématiques en début d'année (régression linéaire bayésienne au niveau de la classe). Les modèles prédictifs de chaque variable incluent toutes les autres à l'exception de celui des indicateurs mobilisés pour construire l'origine socio-économique (l'activité professionnelle des parents la plus prestigieuse, la richesse, le nombre de livres à la maison, et le plus haut niveau d'éducation des parents) dans lesquels l'indice synthétique n'est pas mobilisé. En d'autres termes, les indicateurs sont directement utilisés dans le modèle d'imputation de l'indice synthétique, mais pas le contraire (imputation passive). ampleur, mais néanmoins importante puisqu'elle représente plus d'un cinquième de cette ségrégation totale, une proportion analogue à celle observée par Riegert et Ly (2016) en France. Notons que la ségrégation totale mesurée par un modèle ignorant le niveau classe n'atteint que $21,8 \%$ [16,6; 27,0] (contre $26,3 \%$ dans le modèle complet), ce qui confirme la sous-estimation de la ségrégation totale lorsque seul le niveau école est pris en compte.

L'utilisation de modèles multiniveaux nous permet encore de modéliser les différences socio-économiques entre bassins scolaires (modèle 1). L'inclusion de cette variable diminue la ségrégation entre écoles (et la ségrégation totale) qui reste toutefois importante. L'importance de la ségrégation en FWB est donc liée à des différences entre bassins scolaires, mais elle ne l'est que modérément. La ségrégation ne repose donc pas uniquement sur des disparités régionales, mais bien sur des structurations locales, comme cela a été précédemment exposé. En revanche, assez logiquement, la prise en compte de critère géographique n'a pas d'impact sur la ségrégation entre classes au sein des écoles.

Le modèle 2 nous permet de tester si la ségrégation socio-économique peut se réduire à une ségrégation académique. L'inclusion du niveau en mathématiques et du retard scolaire entraîne une diminution des ségrégations entre écoles et entre classes qui atteignent respectivement $11,6 \%$ et $4,2 \%$. Deux observations s'imposent. Premièrement, la sélection académique explique une partie de la ségrégation socio-démographique entre écoles et entre classes, mais ne l'épuise pas. II reste une ségrégation scolaire proprement socio-économique non réductible à la ségrégation académique. En d'autres termes, à niveau scolaire équivalent, les élèves ont tendance à être regroupés sur la base de critères non scolaires, à savoir dans ce cas-ci, avec d'autres élèves issus du même milieu social. Ainsi, si la ségrégation sociale entre écoles peut s'expliquer en recourant à l'existence d'une stratification académique 
et de marchés socialement contrastés, d'autres mécanismes sont également à l'œuvre. Secondement, les variables modélisées permettent davantage d'expliquer la variance entre écoles que celle entre classes, la part de la dernière dans la variance totale ayant augmenté.

Si la ségrégation socio-économique ne se réduit pas à la ségrégation académique, cela ne veut toutefois pas dire qu'il n'y ait pas de ségrégation académique. Le modèle 3 , où le calcul du VPC est appliqué à notre mesure des compétences mathématiques, confirme l'observation selon laquelle la ségrégation académique est importante et l'est davantage que la ségrégation socio-économique. Nous observons une ségrégation académique importante entre les écoles, mais également entre les classes au sein des écoles.

\section{Discussion}

Plusieurs éléments peuvent être avancés pour expliquer la présence d'une ségrégation sociale, mais également et surtout académique, entre les classes au sein des écoles. Des pratiques de constitution de classes spécifiquement dessinées (notamment par l'organisation de classes de langues moderne ou ancienne) pour l'accueil de populations caractéristiques ont été particulièrement bien documentées en France. Duru-Bellat et Mingat (1997) ont observé qu'au collège, cette pratique n'était pas marginale puisque son application importante concerne une majorité d'établissements. Dans son étude des collèges de banlieue, van Zanten (2001) a mis en évidence le triple rôle de la constitution de «bonnes» classes : attirer et maintenir les «meilleurs» élèves pour lesquels des parcours "protégés» et de qualité sont organisés, gérer l'hétérogénéité des publics et aménager des contextes où les enseignants puissent avoir une pratique professionnelle satisfaisante. En d'autres termes, la séparation des publics sur la base de critères académiques et non académiques peut ainsi répondre à des contraintes réelles découlant d'une convergence entre les stratégies des parents pour s'assurer que leur enfant intègre les meilleures classes et de la direction pour se positionner sur le marché scolaire (Oberti, 2007). Des pressions du corps enseignant peuvent également exister dès lors que le public accueilli détermine les conditions de travail du personnel. Toutefois, cette pratique ne va pas sans poser un certain nombre de problèmes. Alors qu'il n'est pas certain que la pratique permette effectivement le maintien de ces meilleurs élèves, elle a des consé- autres classes étiquetées "mauvaises», mais encore pour le corps professoral puisqu'elle est génératrice de tensions (van Zanten, 2001). Broccolichi et MatheyPierre (2010) confirment la visibilité de ces différences dans certains collèges. Selon les auteurs, la hiérarchisation des classes crée des tensions entre élèves très conscients de cette relégation (d'autant plus lorsque la ségrégation a un caractère visible au travers d'origines sociales ou ethniques distinctes), mais perturbe également le corps enseignant.

Cette image contraste avec ce que les directeurs déclarent dans l'enseignement primaire. Il est vrai que, dans l'enseignement primaire, les parents semblent accepter davantage l'hétérogénéité au sein des classes (van Zanten, 2001). Aux États-Unis, Monk (1987) a interrogé des directeurs d'écoles primaires sur leurs pratiques de constitutions de classes. De ces 17 interviews, il ressort une volonté affirmée de constituer des classes les plus équilibrées possible. Dans l'enseignement primaire en France, la volonté des directions de constituer des classes les plus équilibrées possible a été observée (Leroy-Audouin \& Suchaut, 2005). Tout au plus, les incompatibilités entre élèves sont évitées par leur placement dans des classes séparées. Toutefois, lorsque les chercheurs comparent la composition des classes, ils pointent de légères différences entre classes au regard de l'origine sociale dans certaines écoles et davantage au regard du niveau scolaire moyen de leurs élèves (en mathématiques et en français). De plus, lorsqu'il s'agit d'affecter les élèves à des classes spécifiques comme les classes multiniveaux, des critères comme I'autonomie ou le retard scolaire entrent en jeu (Burns \& Mason, 1998; Leroy-Audouin \& Suchaut, 2005).

La constitution volontaire de classes de niveau (classes académiquement homogènes) n'est pas légalement interdite en FWB, mais le ministère, via ses publications et ses inspecteurs, diffuse un message la déconseillant. Bien que la deuxième secondaire fasse partie du tronc commun, certaines structures de différenciation en termes de carrières scolaires sont toutefois disponibles. Premièrement, des activités complémentaires peuvent être organisées à raison de quatre heures par semaine dès la première année de l'enseignement secondaire. Même s'il peut y avoir une justification organisationnelle forte au regroupement des élèves en classes selon l'activité suivie, une telle pratique n'est toutefois pas obligatoire. Cette disposition rend possible la constitution de classes distinctes sur le plan académique et social sans que cette stratégie doive être explicitement poursuivie (Draelants, Van Ouytsel 
\& Maroy, 2004). Nous pouvons toutefois imaginer que les stratégies explicites des écoles telles qu'observées en France soient également présentes sur le marché belge francophone. L'organisation de classes d'immersion linguistique (où environ la moitié des cours peuvent se donner dans une seconde langue) est une autre possibilité. La fréquentation de ces classes augmente fortement ces dernières années et atteignait 4 à $5 \%$ de la population du premier degré en 2013 (MCF, 2013). Ces classes fournissent un outil important de différenciation des publics entre écoles et au sein de celles-ci. Notons que l'immersion fait partie des critères permettant de classer les demandes d'inscription excédentaires, mais que si le choix de l'immersion dépend des familles, les inscriptions surnuméraires dans les classes en immersion sont départagées selon une procédure standardisée et centralisée.

Le modèle 3 montre clairement que la constitution de classes académiquement homogènes reste légitime sur le terrain. La ségrégation socio-économique sous contrôle du niveau en mathématiques (modèle 2 ) nous indique en outre une tendance à la constitution de classes socialement homogènes, qu'elle soit explicitement organisée par les écoles ou découle d'une auto-sélection des familles dans le choix des activités complémentaires et de l'immersion. II est également possible que la constitution de classes académiquement homogènes se fasse, au moins en partie, sur la base de jugements académiques socialement biaisés.

\section{Conclusion}

Au cours de cet article, nous nous sommes attelés à présenter les différentes mesures de la ségrégation disponibles en FWB et leur apport à la connaissance du phénomène. La littérature a mis en évidence à de nombreuses reprises que cette ségrégation est importante. Davantage marquée au regard des caractéristiques académiques, elle prend sa source dans une organisation particulière de l'enseignement comme machine de tri efficace.

Si le recours à des indices nécessitant de dichotomiser la variable d'intérêt pose problème, c'est davantage la qualité des données qui limite une préhension fine du phénomène. II nous faut rappeler ici la nécessité de disposer de bases de données permettant d'identifier suffisamment de classes par école pour pouvoir mesurer de manière fiable la ségrégation. Sans cela, non seulement la mesure de la ségrégation est déformée, mais sa composante intra-école est surtout ignorée alors qu'elle traduit des mécanismes qui peuvent lui être propres. Nous avons appliqué de façon convaincante une méthode (VPC) à une base de données originale permettant justement cette distinction. Nous confirmons l'importance de la ségrégation et mettons en évidence la part non négligeable de sa composante intra-école.

À la différence d'un quasi-marché, à l'instar de Paris, où la sélection des populations dans les écoles publiques est fortement régulée et où la répartition des élèves entre les classes peut permettre une gestion de I'hétérogénéité des élèves (Maroy \& van Zanten, 2009), la FWB était davantage caractérisée par une faible régulation des inscriptions. Dans ce contexte, nous pouvions nous attendre à observer une ségrégation limitée entre classes. Cette situation a toutefois évolué récemment avec la promulgation des décrets successifs régulant les inscriptions et les changements d'établissement dans le premier degré de l'enseignement secondaire. Dans ce contexte nouveau, il est urgent de pouvoir répliquer ces analyses. Non seulement il se peut que notre mesure reflète une intensification de la ségrégation entre classes, bien que la constitution de classes académiquement hiérarchisées ne soit probablement pas une stratégie nouvelle. Mais il est encore probable qu'une éventuelle diminution de la ségrégation entre écoles soit compensée par une amplification de cette ségrégation intra-école.

II nous reste à souligner les limites de notre mesure de la ségrégation socio-économique. En contrôlant les performances en mathématiques et le retard scolaire, nous avons essayé de tenir compte de la constitution de classes de niveau afin de mesurer une ségrégation uniquement socio-économique non réductible à un regroupement des élèves sur base de leur niveau académique. Trois limites s'imposent néanmoins. La première réside dans l'imperfection de notre mesure des compétences en mathématiques, non seulement parce que notre modélisation ne tient pas compte de l'erreur de mesure, mais encore parce que l'outil mesure partiellement les compétences en mathématiques. Deuxièmement, les mathématiques ne sont qu'une matière, certes principale, parmi l'éventail de matières enseignées à l'école et ne fournissent certainement pas le seul critère définissant l'homogénéité académique de la classe. Troisièmement, l'observation d'une ségrégation socio-économique ne requiert pas une séparation explicite des élèves sur base socioéconomique si l'évaluation du niveau des élèves par les enseignants est imparfaite et biaisée par différents 
stéréotypes. Ceci pourrait se traduire par un décalage entre la constitution des classes homogènes sur la base de notre évaluation et de celle des enseignants. En d'autres termes, une partie de la ségrégation entre classes peut encore être due à la constitution de classes académiquement homogènes.

Julien Danhier

Université libre de Bruxelles, Group for research on Ethnic Relations, Migration and Equality (GERME) jdanhier@ulb.ac.be

Émilie Martin

Université libre de Bruxelles, Group for research on Ethnic Relations, Migration and Equality (GERME)

\section{Bibliographie}

ALLEN R. \& VIGNOLES A. (2007). «What should an index of school segregation measure? ». Oxford Review of Education, vol.33, n5, p.643-668.

ANDRÉ G. (2011). «Les tensions du jugement professoral. Ethnographie des décisions d'orientation scolaire dans les conseils de classe». Éducation et formation, vol.295.

BAYE A. \& DEMEUSE M. (2008). «Indicateurs d'équité éducative. Une analyse de la ségrégation académique et sociale dans les pays européens ». Revue française de pédagogie, $\mathrm{n}^{\circ} 165$, p. 91-103.

BAYE A., DEMEUSE M., MONSEUR C. \& GOFFIN C. (2006). Un ensemble d'indicateurs pour mesurer l'équité des vingt-cinq systèmes éducatifs de l'Union européenne. Liège: Université de Liège, Service de pédagogie théorique et expérimentale.

BAYE A., DEMEUSE M., NICAISE J., MATOUL A., STRAETEN M.-H., BENADUSI L., BOTTANI G., BOVE G., GARCIA DE CORTAZAR M., GIANCOLA O., GORARD S., HUTMACHER W., MEURET D., MORLAIS S., RICOTTA G., SMITH E., TANA-FERRER A. \& VANDENBERGHE V. (2005). L'équité des systèmes éducatifs européens. Un ensemble d'indicateurs. Liège : Université de Liège, Service de pédagogie théorique et expérimentale.

BEATON A. E. \& O'DWYER L. M. (2002). «Separating school, classroom, and student variances and their relationship to socio-economic status ». In D. F. Robitaille, A. E. Beaton (dir.), Secondary analysis of the TIMSS data. Dordrecht : Springer, p. 211-231.

BELL W. (1954). «A Probability Model for the Measurement of Ecological Segregation». Social Forces, vol.32, n4, p. 357-364.

BOONE S. \& VAN HOUTTE M. (2013). «Why are teacher recommendations at the transition from primary to secondary education socially biased? A mixed-methods research». British Journal of Sociology of Education, vol.34, n¹, p.20-38.

BRESSOUX P. (1995). «Les effets du contexte scolaire sur les acquisitions des élèves : effet-école et effets-classes en
Alejandra Alarcon-Henriquez

Université libre de Bruxelles, Group for research on Ethnic Relations, Migration and Equality (GERME)

Rob Kaelen

Université libre de Bruxelles, Group for research on Ethnic Relations, Migration and Equality (GERME)

Dirk Jacobs

Université libre de Bruxelles, Group for research on Ethnic Relations, Migration and Equality (GERME)

lecture». Revue française de sociologie, vol. $36, \mathrm{n}^{\circ} 2$, p. 273-294.

BROCCOLICHI S. \& MATHEY-PIERRE C. (2010). «Un durcissement des oppositions entre "bons" et "mauvais"». In S. Broccolichi, C. Ben Ayed \& D. Trancart, École : les pièges de la concurrence. Paris : La Découverte, p.166-179.

BROWNE W. J. \& DRAPER D. (2006). «A comparison of Bayesian and likelihood-based methods for fitting multilevel models ». Bayesian Analysis, vol. 1, n³, p.473-514.

BURNS R. B. \& MASON D. A. (1998). «Class Formation and Composition in Elementary Schools ». American Educational Research Journal, vol.35, n², p.739-772.

CLOTFELTER C. T., LADD H. F. \& VIGDOR J. L. (2003). «Segregation and Resegregation in North Carolina's Public School Classrooms». North Carolina Law Review, vol. 81, p. 1463.

CONGER D. (2005). «Within-School Segregation in an Urban School District ». Educational Evaluation and Policy Analysis, vol.27, n³, p.225-244.

CORTESE C. F., FALK R. F. \& COHEN J. K. (1976). «Further Considerations on the Methodological Analysis of Segregation Indices». American Sociological Review, vol.41, $\mathrm{n}^{\circ} 4$, p. 630-637.

CRAHAY M. (2000). L'école peut-elle être juste et efficace? De l'égalité des chances à l'égalité des acquis. Bruxelles : De Boeck.

CRAHAY M. (2007). Peut-on lutter contre l'échec scolaire? Bruxelles : De Boeck, $3^{\mathrm{e}}$ éd.

DANHIER J. (2017). «How Big Is the Handicap for Disadvantaged Pupils in Segregated Schooling Systems?». British Journal of Educational Studies, vol.66, n³, p.341-364.

DANHIER J. \& DENY E. (2009). Une mesure de la mixité socioéconomique dans les implantations de la Communauté française. Papier de travail du service des statistiques de I'ETNIC.

DANHIER J. \& DEVLEESHOUWER P. (2017). «School choice 
and local embeddedness in Brussels: the neighbourhood effect assessed through administrative files». Belgeo. Revue belge de géographie, $\mathrm{n}^{\circ} 2-3$.

DANHIER J. \& FRIANT N. (2016). «Measuring local socioeconomic segregation in schools with an international largescale survey: the case of PISA in the Belgian French community ». In J. Danhier, Little Matthew has also chosen the wrong school: Secondary analyses of compositional effect in a segregated educational system. Thèse de doctorat, sciences politiques et sociales, Université de Bruxelles, p.113-133.

DANHIER J., JACOBS D., DEVLEESHOUWER P., MARTIN É. \& ALARCON-HENRIQUEZ A. (2014). Vers des écoles de qualité pour tous? Analyse des résultats à l'enquête PISA 2012 en Flandre et en Fédération Wallonie-Bruxelles. Bruxelles : Fondation Roi Baudouin.

DANHIER J. \& MARTIN É. (2014). «Comparing Compositional Effects in Two Education Systems: The Case of the Belgian Communities». British Journal of Educational Studies, vol. 62, n², p. 171-189.

DELVAUX B. (2000). «Orientation et redoublement : recomposition de deux outils de gestion des trajectoires scolaires». In G. Bajoit (dir.), Jeunesse et société: la socialisation des jeunes dans un monde en mutation. Bruxelles : De Boeck.

DELVAUX B. (2003). "La ségrégation scolaire, phénomène avéré, mais mal connu : apports et limites des données disponibles en Belgique francophone ». Cahiers du Service de pédagogie expérimentale (Ulg), vol.15-16, p.113-124.

DELVAUX B. (2005). «Ségrégation scolaire dans un contexte de libre choix et de ségrégation résidentielle». In M. Demeuse, A. Baye, M.-H. Straeten, J. Nicaise \& A. Matoul (dir.), Vers une école juste et efficace. Bruxelles : De Boeck, p. 275-295.

DELVAUX B., DEMEUSE M., DUPRIEZ V., FAGNANT A., GUISSET C., LAFONTAINE D., MARISSAL P. \& MAROY C. (2005). Les bassins scolaires : de l'idée au projet. Propositions relatives aux domaines d'intervention, aux instances et aux territoires. Rapport à la Communauté française de l'étude intitulée «Définition, cartographie, rôles et modes de régulation des bassins scolaires".

DELVAUX B. \& JOSEPH M. (2006). «Hiérarchie scolaire et compétition entre écoles : le cas d'un espace local belge». Revue française de pédagogie, $\mathrm{n}^{\circ} 156, \mathrm{p} .19-27$.

DELVAUX B. \& MAROY C. (2009). «Débat sur la régulation des inscriptions scolaires en Belgique francophone : où se situent les désaccords? ». Les Cahiers de recherche du Girsef, vol. 68 .

DELVAUX B. \& SERHADLIOGLU E. (2014). «La ségrégation scolaire, reflet déformé de la ségrégation urbaine. Différenciation des milieux de vie des enfants bruxellois. ». Les Cahiers de recherche du Girsef, vol. 100.

DEMEUSE M. (2002). Analyse critique des fondements de l'attribution des moyens destinés à la politique de discrimination positive en matière d'enseignement en Communauté française de Belgique. Thèse de doctorat, sciences psychologiques, Université de Liège.
DEMEUSE M. \& BAYE A. (2008). «Mesurer et comparer l'équité des systèmes éducatifs en Europe». Éducation et formation, vol.78, p.137-149.

DEMEUSE M., DEROBERTMASURE A., FRIANT N., HERREMANS T., MONSEUR C., UYTTENDAELE S. \& VERDALE N. (2007). Étude exploratoire sur la mise en œuvre de nouvelles mesures visant à lutter contre les phénomènes de ségrégation scolaire et d'inéquité au sein du système éducatif de la Communauté française de Belgique. Bruxelles : Gouvernement de la Communauté française de Belgique (unpublished research report).

DEMEUSE M. \& FRIANT N. (2010). «School segregation in the French Community of Belgium ». In J. Bakker, E. Denessen, D. Peters \& G. Walraven (dir.), International perspectives on countering school segregation. Antwerpen : Garant, p.169-187.

DEVLEESHOUWER P. \& REA A. (2011). «Justification des différenciations scolaires par les acteurs de l'enseignement ». Éducation comparée, Nouvelle série, vol.6, p.49-68.

DRAELANTS H. (2006). «Le redoublement est moins un problème qu'une solution. Comprendre l'attachement social au redoublement en Belgique francophone». Les Cahiers de recherche du Girsef, vol. 52 .

DRAELANTS H. (2018). «Le redoublement est-il vraiment moins efficace que la promotion automatique? Une évidence à réinterroger ». Les Cahiers de recherche du Girsef, vol. 113.

DRAELANTS H. \& BALLATORE M. (2015). «Capital culturel et reproduction scolaire. Un bilan critique». Revue française de pédagogie, $\mathrm{n}^{\circ} 186, \mathrm{p} .115-142$.

DRAELANTS H., VAN OUYTSEL A. \& MAROY C. (2004). "Logiques locales d'établissements et mise en œuvre d'une réforme : le cas de l'organisation des années complémentaires dans deux établissements contrastés ». In M. Frenay \& C. Maroy (dir.), L'École, six ans après le décret «missions». Louvain-la-Neuve : Presses universitaires de Louvain, p.209-235.

DUMAY X. \& DUPRIEZ V. (2008). «Does the school composition effect matter? Evidence from Belgian data ». British Journal of Educational Studies, vol.56, n 4, p. 440-477.

DUNCAN O. D. (1961). Statistical geography: Problems in analyzing a real data. New York : Free Press.

DUNCAN O. D. \& DUNCAN B. (1955). «A Methodological Analysis of Segregation Indexes». American Sociological Review, vol.20, n², p.210-217.

DUPONT V. \& LAFONTAINE D. (2011). «Les choix d'études supérieures sont-ils liés à l'établissement secondaire fréquenté? ». Schweizerische Zeitschrift für Bildungswissenschaften, vol.3, $n^{\circ} 33$, p. 461-478.

DUPRIEZ V. \& VANDENBERGHE V. (2004). «L'école en Communauté française de Belgique : de quelle inégalité parlons-nous? ». Les Cahiers de recherche du Girsef, vol.27.

DURU-BELLAT M. \& MINGAT A. (1997). «La constitution de classes de niveau dans les collèges : les effets pervers d'une pratique à visée égalisatrice». Revue française de sociologie, vol.38, n4, p.759-789. 
ERIKSON R. (1984). «Social Class of Men, Women and Families». Sociology, vol.18, n4, p.500-514.

FERRARA M. \& FRIANT N. (2014). «Les représentations sociales des élèves du premier et du dernier degré de l'enseignement secondaire en Belgique francophone par rapport aux différentes filières». L'Orientation scolaire et professionnelle, $\mathrm{n}^{\circ} 43-4$.

FRANKEL D. M. \& VOLIJ O. (2011). «Measuring school segregation ». Journal of Economic Theory, vol.146, n ${ }^{\circ}$, p.1-38.

FRIANT N. (2015). «Choix de l'école et ségrégations scolaires dans un espace local en Belgique francophone». Communication au colloque international Inégalités éducatives et espaces de vie, organisé à Rennes les 10 et 11 septembre 2015.

GANZEBOOM H. B. (2010). «A new International Socio-Economic Index (ISEI) of occupational status for the International Standard Classification of Occupation 2008 (ISCO08) constructed with data from the ISSP 2002-2007». Paper presented at Annual Conference of International Social Survey Programme, Lisbonne.

GELMAN A., CARLIN J. B., STERN H. S. \& RUBIN D. B. (2003). Bayesian Data Analysis. Boca Raton : Chapman and Hall/ $\mathrm{CRC}, 2^{\mathrm{e}}$ éd.

GOOS M., VAN DAMME J., ONGHENA P., PETRY K. \& DE BILDE J. (2013). «First-grade retention in the Flemish educational context: Effects on children's academic growth, psychosocial growth, and school career throughout primary education». Journal of school psychology, vol.51, n³, p.323-347.

GORARD S. \& TAYLOR C. (2002). «What is Segregation? A Comparison of Measures in Terms of "Strong" and "Weak" Compositional Invariance». Sociology, vol.36, $n^{\circ} 4$, p. 875-895.

GRISAY A. (1984). «Les mirages de l'évaluation scolaire (1). Rendement en français, notes et échecs à l'école primaire». Revue de la Direction générale de l'organisation des études, vol.19, n5, p.29-42.

GRISAY A. (1995). «Trop de disparités d'une école à l'autre au début de l'enseignement secondaire en Communauté française de Belgique». Pilotinfo, n4, p. 1-7.

GROVES R. M., FLOYD J. F. J., COUPER M. P., LEPKOWSKI J. M., SINGER E. \& TOURANGEAU R. (2009). Survey Methodology. Hoboken : John Wiley \& Sons, $2^{\mathrm{e}}$ éd.

HINDRIKS J. \& LAMY G. (2013). «Retour à l'école, retour à la ségrégation?» Itinera Insitute Analyse, $\mathrm{n}^{\circ} 5$.

HINDRIKS J., VERSCHELDE M., RAYP G. \& SCHOORS K. (2009). "Analyse des disparités régionales d'éducation en Belgique». Actes du $18^{e}$ Congrès des Économistes belges de langue française "Quel État pour quelles performances économiques?» Université libre de Bruxelles : CIFoP, p.71-87.

HOOPER D., COUGHLAN J. \& MULLEN M. (2008). «Structural equation modelling: Guidelines for determining model fit ». Electronic Journal of Business Research Methods, vol. 6, $\mathrm{n}^{\circ} 1, \mathrm{p} .53-60$.

HU L. \& BENTLER P. M. (1999). «Cutoff criteria for fit indexes in covariance structure analysis: Conventional criteria ver- sus new alternatives». Structural Equation Modeling: A Multidisciplinary Journal, vol. 6, nº1, p.1-55.

HUTCHENS R. (2001). «Numerical measures of segregation: desirable properties and their implications». Mathematical Social Sciences, vol. 42, $n^{\circ} 1$, p. 13-29.

JACOBS D., MARTIN E., ALARCON-HENRIQUEZ A., DANHIER J. \& KAELEN R. (2017). Equal opportunities in educational systems with high levels of social and ethnic segregation (Equop project) - Codebook. Bruxelles : Université libre de Bruxelles.

JACOBS D. \& REA A. (2005). «Construction et importation des classements ethniques». Revue européenne des migrations internationales, vol.21, n², p.35-59.

JAMES D. R. \& TAEUBER K. E. (1985). «Measures of Segregation ». Sociological Methodology, vol.15, p.1-32.

JENKINS S. P., MICKLEWRIGHT J. \& SCHNEPF S. V. (2008). "Social segregation in secondary schools: how does England compare with other countries? ». Oxford Review of Education, vol.34, $\mathrm{n}^{\circ} 1$, p. 21-37.

KALOGRIDES D. \& LOEB S. (2013). «Different Teachers, Different Peers: The Magnitude of Student Sorting Within Schools». Educational Researcher, vol. 42, n 6, p. 304-316.

KESKPAIK S. \& ROCHERT. (2011). «La mesure de l'équité dans PISA». Memoriam, n`80, p. 69.

LAFONTAINE D. (1996). Performances en lecture et contexte éducatif : enquête internationale menée auprès d'élèves de 9 et 14 ans. Bruxelles : De Boeck.

LAFONTAINE D. \& MONSEUR C. (2011). «Quasi-marché, mécanismes de ségrégation sociale et académique. Une approche comparative». Éducation comparée, Nouvelle série, vol.6, p.69-90.

LE GRAND J. (1991). «Quasi-markets and social policy». The Economic Journal, p. 1256-1267.

LEROY-AUDOUIN C. \& SUCHAUT B. (2005). «À chaque classe ses élèves : procédures et critères d'affectation à l'école élémentaire». Revue française de pédagogie, $\mathrm{n}^{\circ} 152$, p. 89-105.

MAAS C. J. M. \& HOX J. (2005). «Sufficient Sample Sizes for Multilevel Modeling». Methodology: European Journal of Research Methods for the Behavioral and Social Sciences, vol.1, n³, p. 86-92.

MACCALLUM R. C., ZHANG S., PREACHER K. J. \& RUCKER D. D. (2002). «On the practice of dichotomization of quantitative variables». Psychological Methods, vol.7, n¹, p.19-40.

MARISSAL P. (2014). "La ségrégation entre écoles maternelles. Inégalités entre implantations scolaires : les inégalités sociales entre quartiers ont trop bon dos». Éducation et formation, $\mathrm{n}^{\circ} \mathrm{e}-302$.

MAROY C. \& DUPRIEZ V. (2000). «La régulation dans les systèmes scolaires : proposition théorique et analyse du cadre structurel en Belgique francophone». Revue française de pédagogie, n¹30, p.73-87.

MAROY C. \& van ZANTEN A. (2009). «Regulation and competition among schools in six European localities». Sociologie du travail, vol.51, n¹, p. e67-e79. 
MARTIN M. O., MULLIS I. V. S. \& FOY P. (2011). «Age distribution and reading achievement configurations among fourth-grade students in PIRLS 2006 ». IERI Monographic Series: Issues and Methodologies in Large-Scale Assessments, vol. 4, p. 9-33.

MCF (2005). Contrat pour l'école. 10 priorités pour nos enfants. Bruxelles : Gouvernement de la Communauté française de Belgique.

MCF (2010). Statistiques de l'enseignement de plein exercice et budget des dépenses d'enseignement : Annuaire 2008-2009. Bruxelles: Ministère de la Communauté française.

MCF (2013). Indicateurs de l'enseignement 2013. Bruxelles : Ministère de la Communauté française.

MCF (2014). Rapport de la Comission de Pilotage relatif au décret inscription. Bruxelles : Ministère de la Communauté française.

MCF (2016). Indicateurs de l'enseignement 2015. Bruxelles : Ministère de la Communauté française.

MONK D. H. (1987). «Assigning Elementary Pupils to Their Teachers». Elementary School Journal, vol.88, $\mathrm{n}^{\circ} 2$, p.167-187.

MONSEUR C. \& DEMEUSE M. (2001). «Gérer l'hétérogénéité des élèves. Méthodes de regroupement des élèves dans l'enseignement obligatoire». Cahiers du Service de Pédagogie expérimentale, vol.7-8, p.25-52.

MONSEUR C. \& LAFONTAINE D. (2005). «Methodological Issues Raised by Equity Indicators Derived from Multilevel Analyses». International Workshop on "Normative and Quantitative Analysis of Educational Inequalities».

OBERTI M. (2007). L'école dans la ville. Ségrégation-mixité-carte scolaire. Paris : Presses de Sciences Po.

OECD (2004). Learning for tomorrow's world: First results from PISA 2003. Paris : OECD Publishing.

OECD (2007). PISA 2006: Science Competencies for Tomorrow's World (Volume 1: Analysis). Paris : OECD Publishing.

OECD (2010). PISA 2009 Results: Overcoming social background-Equity in learning opportunities and outcomes (Volume II). Paris : OECD Publishing.

OECD (2014). PISA 2012 Results: What Makes Schools Successful? Paris : OECD Publishing.

OPDENAKKER M.-C. \& VAN DAMME J. (2000). «The importance of identifying levels in multilevel analysis: an illustration of the effects of ignoring the top or intermediate levels in school effectiveness research ». School Effectiveness and School Improvement, vol.11, p.103-130.
PFEFFERMANN D., SKINNER C. J., HOLMES D. J., GOLDSTEIN H. \& RASBASH J. (1998). «Weighting for unequal selection probabilities in multilevel models». Journal of the Royal Statistical Society: Series B (Statistical Methodology), vol.60, $\mathrm{n}^{\circ} 1$, p. 23-40.

RANSOM M. R. (2000). «Sampling Distributions of Segregation Indexes». Sociological Methods \& Research, vol.28, $\mathrm{n}^{\circ} 4$, p. 454-475.

RIEGERT A. \& LY S. T. (2016). «Inégalités scolaires, ségrégation et effets de pairs ». In A. Riegert, Inégalités scolaires, ségrégation et effets de pairs. Thèse de doctorat, sciences économiques, EHESS, p.107-156.

RUBIN D. B. (1987). Multiple imputation for nonresponse in surveys. New York : Wiley.

RYELANDT N. (2013). Les décrets «inscriptions» et "mixité sociale » de la Communauté française. Bruxelles : CRISP.

TABACHNICK B. G. \& FIDELL L. S. (2007). Using multivariate statistics. Boston : Allyn and Bacon, $5^{\mathrm{e}}$ éd.

VAN BUUREN S. \& GROOTHUIS-OUDSHOORN K. (2011). «MICE: Multivariate imputation by chained equations in $\mathrm{R} »$. Journal of statistical software, vol.45, $\mathrm{n}^{\circ} 3$.

VAN ZANTEN A. (2001). L'école de la périphérie. Paris : PUF.

VANDENBERGHE V. (1996). Functioning and regulation of educational quasi-markets. Louvain-la-Neuve : Ciaco.

VERSCHELDE M., HINDRIKS J., RAYP G. \& SCHOORS K. (2010). Explaining social segregation in Belgium: an index decomposition approach. UGent, Working Paper 10.69.

WHITE K. R. (1982). «The relation between socioeconomic status and academic achievement». Psychological Bulletin, vol. 91, n³, p.461-481.

WHITE M. J. (1986). «Segregation and Diversity Measures in Population Distribution». Population Index, vol.52, n², p.198-221.

WILLMS J. D. \& PATERSON L. (1995). «A multilevel model for community segregation ». The Journal of Mathematical Sociology, vol.20, n¹, p.23-40.

YANG Y. \& GUSTAFSSON J.-E. (2004). «Measuring Socioeconomic Status at Individual and Collective Levels ». Educational Research and Evaluation, vol. 10, n³, p.259-288.

ZHOU X. \& REITER J. P. (2010). «A Note on Bayesian Inference After Multiple Imputation ». The American Statistician, vol. $64, n^{\circ} 2$, p. 159-163. 


\section{Annexes}

Tableau 2. Mesures de la ségrégation entre écoles en Fédération Wallonie-Bruxelles

\begin{tabular}{|c|c|c|c|c|c|c|}
\hline Article & $\begin{array}{l}\text { Indice } \\
\text { (Seuil \%) }\end{array}$ & Indicateur & Données & Valeur & Rang & IC \\
\hline \multirow[t]{3}{*}{ Baye, Demeuse, Nicaise et al., 2005} & GS (10) & Mathématiques & PISA 2000 & 0,61 & $18 / 19$ & \\
\hline & GS (10) & HISEI & PISA 2000 & 0,35 & $16 / 19$ & \\
\hline & GS (10) & Mathématiques & TIMMS 1995 & 0,41 & $12 / 17$ & \\
\hline \multirow[t]{2}{*}{ Baye, Demeuse, Monseur et al., 2006} & GS (10) & HISEI & PISA 2003 & $0,38^{*}$ & $22 / 24$ & \\
\hline & GS (10) & Mathématiques & PISA 2003 & $0,59 *$ & $21 / 24$ & \\
\hline \multirow[t]{2}{*}{ Baye \& Demeuse, 2008} & GS (10) & Mathématiques & PISA 2006 & $0,58^{*}$ & $24 / 32$ & \\
\hline & GS (10) & HISEI & PISA 2006 & $0,34^{*}$ & $20 / 32$ & \\
\hline Danhier \& Deny, 2009 & VPC & ISE & CMP 2008 & 0,44 & & \\
\hline \multirow[t]{4}{*}{ Danhier \& Friant, 2016} & GS (15) & ESCS & PISA 2012 & 0,34 & & $0,30-0,37$ \\
\hline & GS (15) & ISE & CMP 2012 & 0,47 & & \\
\hline & VPC & ESCS & PISA 2012 & 0,26 & & $0,21-0,27$ \\
\hline & VPC & ISE & CMP 2012 & 0,40 & & \\
\hline \multirow{3}{*}{$\begin{array}{l}\text { Danhier, Jacobs, Devleeshouwer } \\
\text { et al., } 2014\end{array}$} & GS (15) & ESCS & PISA 2012 & 0,36 & $16 / 22$ & $0,32-0,39$ \\
\hline & GS (15) & Mathématiques & PISA 2012 & 0,42 & $15 / 22$ & $0,39-0,46$ \\
\hline & VPC & Mathématiques & PISA 2012 & 0,56 & $19 / 22$ & \\
\hline \multirow[t]{2}{*}{ Dupriez \& Vandenberghe, 2004} & $\mathrm{D}(25)$ & Mathématiques & PISA 2000 & 0,59 & $19 / 21$ & \\
\hline & $\mathrm{D}(25)$ & HISEI & PISA 2000 & 0,49 & $21 / 21$ & \\
\hline Hindriks, Verschelde, Rayp et al., 2009 & $\mathrm{D}(20)$ & ESCS & PISA 2006 & 0,43 & $29 / 32$ & $0,38-0,49$ \\
\hline \multirow{2}{*}{$\begin{array}{l}\text { Verschelde, Hindriks, Rayp, } \\
\text { \& Schoors, } 2010\end{array}$} & $H(50)$ & ESCS & PISA 2006 & 0,18 & & $0,14-0,22$ \\
\hline & $\mathrm{D}(50)$ & ESCS & PISA 2006 & 0,44 & & $0,39-0,50$ \\
\hline Hindriks \& Lamy, 2013 & $\mathrm{D}(50)$ & ESCS & PISA 2009 & 0,46 & $33 / 36$ & \\
\hline Jenkins, Micklewright, \& Schnepf, 2008 & $\mathrm{D}(50)$ & HISEI & PISA 2000-03 & $0,40^{*}$ & $26 / 27$ & $0,38-0,43$ \\
\hline Grisay, 1995 & VPC & Mathématiques & IEA $1981(2 S)$ & 0,54 & $11 / 13$ & \\
\hline \multirow[t]{4}{*}{ Lafontaine, 1996} & VPC & Lecture & IEA 1991 (4P) & 0,16 & $11 / 19$ & \\
\hline & VPC & Lecture & IEA 1991 (2S) & 0,38 & $13 / 19$ & \\
\hline & VPC & Possessions & IEA 1991 (4P) & 0,19 & & \\
\hline & VPC & Possessions & IEA 1991 (2S) & 0,21 & & \\
\hline MCF, 2014 & $\mathrm{D}(20,4)$ & ISE & CMP 2013 & 0,17 & & \\
\hline \multirow[t]{2}{*}{ OECD, 2004} & VPC & Mathématiques & PISA 2003 & $0,46^{*}$ & $22 / 30$ & \\
\hline & VPC & ESCS & PISA 2003 & $0,32^{*}$ & $23 / 30$ & \\
\hline \multirow[t]{2}{*}{ OECD, 2007} & VPC & Mathématiques & PISA 2006 & $0,52^{*}$ & $23 / 31$ & \\
\hline & VPC & ESCS & PISA 2006 & $0,27^{*}$ & $21 / 31$ & \\
\hline \multirow[t]{2}{*}{ OECD, 2010} & VPC & Lecture & PISA 2009 & $0,53^{*}$ & $25 / 33$ & \\
\hline & VPC & ESCS & PISA 2009 & $0,30^{*}$ & $27 / 33$ & \\
\hline \multirow[t]{2}{*}{ OECD, 2014} & VPC & Mathématiques & PISA 2012 & $0,51^{*}$ & $25 / 33$ & \\
\hline & VPC & ESCS & PISA 2012 & $0,28^{*}$ & $26 / 33$ & \\
\hline
\end{tabular}


Le tableau 2 présente les valeurs des différents indices calculés en FWB, leurs intervalles de confiance lorsqu'ils ont été calculés, ainsi que le rang occupé par la FWB lorsqu'il s'agit de comparaisons internationales. Notons qu'un astérisque est accolé à la valeur de la mesure si les auteurs n'ont mesuré la ségrégation que pour la Belgique, sans distinguer les communautés linguistiques.

Figure 2. VPC pour les niveaux école, classe et élève (imputations et combinaisons)

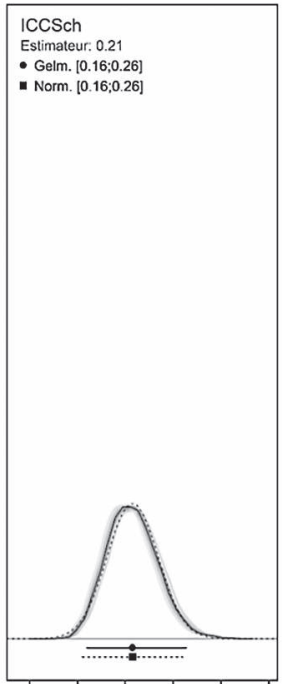

$\begin{array}{llllll}0.10 & 0.15 & 0.20 & 0.25 & 0.30 & 0.35\end{array}$

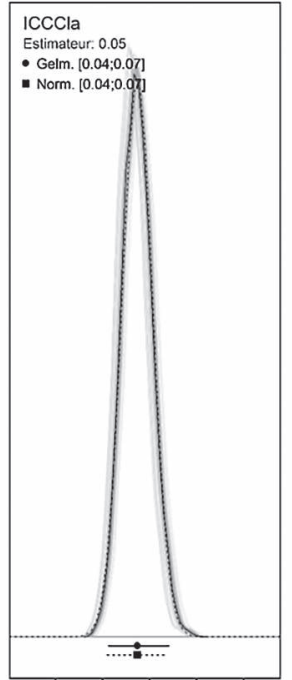

$\begin{array}{lllll}0.02 & 0.04 & 0.06 & 0.08 & 0.10\end{array}$

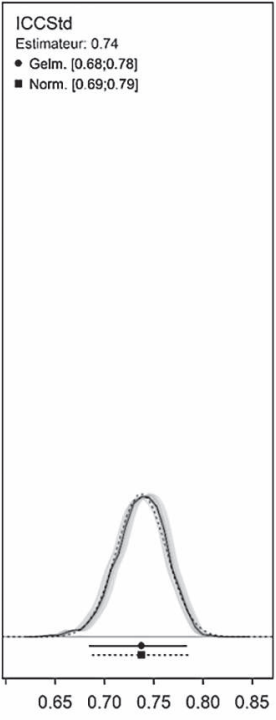

Deux techniques nous ont permis de combiner les distributions postérieures du VPC. Premièrement, postulant que la distribution du VPC est normale, nous avons combiné les variances du VPC de chaque imputation selon les règles classiques de Rubin (1987) et calculé ensuite un intervalle de confiance. Secondement, nous avons mixé les distributions postérieures du VPC de chaque imputation comme suggéré par Gelman, Carlin, Stern et alii (2003) et validé par Zhou et Reiter (2010), et obtenu un intervalle de crédibilité sur la base de cette distribution. La figure 2 représente les distributions des indices VPC (modèle 0) pour chaque imputation (courbes claires) ainsi que les distributions obtenues selon la méthode de Gelman (courbe et ligne noire continue) et celle de Rubin (courbe et ligne noire pointillée). La distribution de nos indices se rapprochant d'une distribution normale, les deux méthodes fournissent des intervalles proches. Nous reporterons uniquement les résultats de la méthode de Gelman, plus cohérente au regard de l'approche globale. 
